\title{
Multipore zeolites: synthesis and catalytic applications
}

Manuel Moliner, Cristina Martínez, Avelino Corma*

Instituto de Tecnología Química, Universidad Politécnica de Valencia, Consejo Superior de Investigaciones Científicas (UPV-CSIC), Valencia, 46022, Spain

*Corresponding author: E-mail address: acorma@itq.upv.es 


\begin{abstract}
In the last years, important efforts are being performed to synthesize multipore zeolites, which contain channels of different dimensions within the same crystalline structure. This is a very attractive subject, since the presence of pores of different sizes would favor the preferential diffusion of reactants and products through those different channel systems, allowing unique catalytic activities for specific chemical processes. In the present review, we describe the most attractive achievements on the rational synthesis of multipore zeolites, containing from small to extra-large pores, and the improvements reported on relevant chemical processes when these multipore zeolites have been used as catalysts.
\end{abstract}

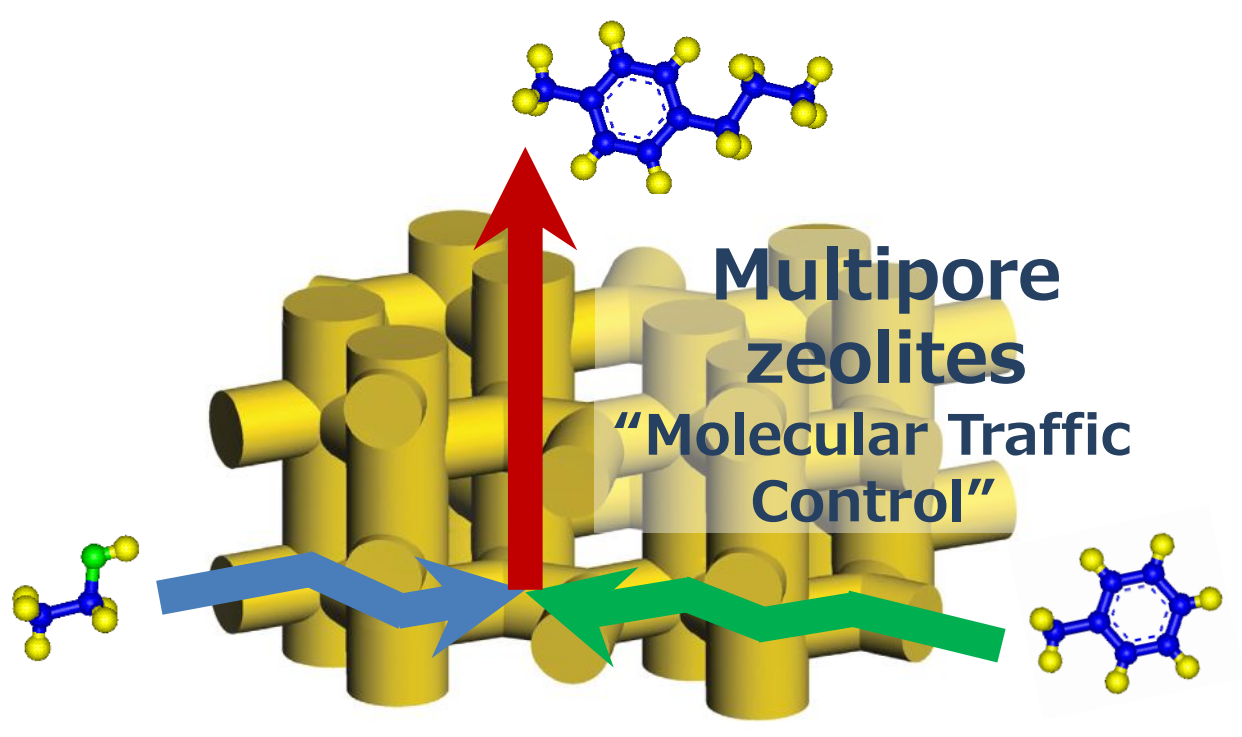




\section{1.- Introduction}

Zeolites or related materials are crystalline microporous oxide materials with welldefined pores and cavities of molecular dimensions ( 3-12 $⿱ 2)$ within their structure. ${ }^{1}$ The ability of tailoring their chemical composition and pore topology has allowed a wide range of industrial applications, particularly as catalysts in oil refining, petrochemicals, and chemicals. ${ }^{2}$

The most extended chemical composition of zeolites is as silicoaluminates. ${ }^{3}$ Zeolitic silicoaluminates usually show high Brønsted acidity due to the presence of protons that are balancing the negative charges generated by the insertion of trivalent Al atoms in tetrahedral coordination into the zeolitic framework. ${ }^{4}$ These acid materials are applied as efficient catalysts in industrial processes demanding Brønsted acidity, and it is especially relevant their use in oil refining and petrochemistry, e.g. isomerization, alkylation or cracking and hydrocracking of hydrocarbons. ${ }^{2 a, c, d, 5}$ On the other hand, the isomorphic substitution of several isolated metals, such as titanium, tin, or zirconium, among others, into the zeolitic framework of high-silica materials, allows the preparation of Lewis-acid containing zeolites. ${ }^{6}$ These materials have been applied as very selective catalysts for relevant oxidation reactions, especially in the field of fine chemicals ${ }^{6 a, 7}$ and, more recently, their potential application in biomass transformation processes is being extensively studied. ${ }^{8}$ Finally, the preparation of zeotypes in silicoaluminophosphate (SAPO) or metalloaluminophosphate (MeAIPO, where Me can be Co, $\mathrm{V}, \mathrm{Ti}$, among others) forms, has allowed the application of new selective catalysts in chemical processes requiring Brønsted acid or redox sites, respectively. ${ }^{9}$

In addition to the chemical composition, the pore dimensions and topology defining the crystalline structure is another important feature of zeolites. This is a key issue since most of the chemical reactions involved in a catalytic process should take place on the active sites within the internal pores and cavities of the zeolite. Therefore, the size, shape and dimensionality of the pores/cavities present in the zeolite structures will be determinant for their application as catalysts in particular chemical processes. ${ }^{4,10}$ Indeed, the unique microporous nature of zeolites with well-defined and regular pores of these materials, allows controlling the accessibility of reactants to the 
active sites and reactivity control by shape-selectivity on reactants, intermediate transition-states or products. ${ }^{11}$

The International Zeolite Association (IZA) recognizes 213 different zeolitic structures, which can be classified depending on the size of the openings that define the pores (determined by the number of heteroatoms defining the pores) or the number of dimensions through which a molecule can diffuse within the crystals (see Figure 1). In general, two-dimensional or three-dimensional zeolites with large or extra-large pores (> $7 \AA$ ) allow processing bulkier reactants and products, while the reduction of the zeolite dimensions and/or pore sizes results in hindered diffusion paths or severe steric restrictions to the organic molecules involved in the catalytic process. ${ }^{10 a, 12}$ However, these limitations can turn into a virtue when small molecules have to be processed. For instance, very attractive industrial applications using small pore zeolites $(<4 \AA$ ) have gained importance along the last decade, such as methanol-to-olefins (MTO) or selective catalytic reduction (SCR) of NOx. ${ }^{5}$

If the zeolites accepted by the IZA are analyzed, it can be observed that several structures show interconnected pores of different size (see Table 1). The pore topologies of these so called multipore zeolites include the combination of small and medium, small and large, medium and large, extra-large and medium, or extra-large and large pores (see Table 1). The presence of pores of different sizes could favor the preferential diffusion of reactants and products through different channel systems, establishing improved activities and selectivities for specific catalytic processes owing to a potential "Molecular Traffic Control (MTC)", as introduced by Derouane et al. in 1980. ${ }^{13}$ In their work, the authors claimed a new shape selectivity effect that can occur in the intracrystalline volume of multipore zeolites, where reactant molecules can preferentially diffuse through a given channel while the products can diffuse out through another channel system, preventing in this way counterdiffusion limitations. The MTC was based on the selective adsorption results of different hydrocarbons on MFI zeolites. ${ }^{13}$ Although the concept generated some controversy in the early years, ${ }^{14}$ several theoretical reports have demonstrated that reactivity enhancement may occur by MTC effects. ${ }^{15}$

Besides the MTC effect described for molecular sieves presenting interconnected pores of different size, some of these materials can present a different population of 
framework Al and, consequently, a different population of active sites in the different channels. They also show very specific cavities or "pockets" in the intersection of these different channels. ${ }^{16}$ The form and shape of these cavities and/or the population of the active sites in precise positions within these cavities can help discriminating among different possible reaction transition states, or can have a direct influence on the entropy of activation, making the catalyst more active and selective for a particular

reaction. ${ }^{16}$ Recently, one of the most remarkable examples on active site specificity in zeolites has been described for the low-temperature carbonylation of dimethyleter (DME) with CO using multipore acidic zeolites with particular cavities/pockets. ${ }^{16 c, 17}$

In the present manuscript, we would like to review the most interesting zeolites and related materials presenting multipore structures, including their synthesis methodologies and their properties as catalysts. On one hand, we will make special emphasis on those synthetic procedures that have been rationalized by selecting the appropriate conditions (i.e. organic and/or inorganic structure directing agents or postsynthetic treatments, among others) to direct the synthesis of zeolites with a particular multipore framework, or with a preferential active site location within the pores/cavities. On the other hand, we will also highlight some catalytic applications where the use of multipore zeolites has allowed improving the catalytic activity and/or selectivity for particular chemical processes. The present review will be divided according to the type of pores defining the multipore zeolites, classifying them as molecular sieves with small and medium, small and large, medium and large, and extra-large and smaller pores.

\section{2.- Zeolites with small and medium pores}

As it can be seen in Table 1, several zeolites have been synthesized containing interconnected medium and small pores in their structure. From all these multipore zeolites with medium and small openings, we have selected the materials presenting either more rationalized synthesis procedures, or interesting catalytic applications based on their multipore structures.

\section{1.- Ferrierite (FER)}


The structure of FER is formed by two-dimensional interconnected straight pores, including a 10-ring channel with openings of $4.2 \times 5.4 \AA$ along the [001] direction, and an 8-ring channel with openings of $3.5 \times 4.8 \AA$ along the [010] direction. This material is probably the most attractive zeolite containing interconnected medium and small pores in the same structure. Indeed, FER can be prepared under very broad synthesis conditions, ranging from high silica (even pure silica) when different organic structure directing agents (OSDAs) are used, ${ }^{18}$ to very low Si/Al ratios when the synthesis is carried out in the absence of OSDAs. ${ }^{19}$ In the first synthesis description of FER, it was named Sr-D zeolite as reported by Barrer et al. in 1964, and it was prepared in the absence of organic molecules as a strontium-containing silicoaluminate. ${ }^{20}$ Along the last decade, different groups have been able to synthesize FER with a Si/Al ratio of almost 15 under OSDA-free synthesis conditions by means of a seeding methodology. ${ }^{21}$ This is a very attractive advantage in comparison to other reported OSDA-free zeolites that have been synthesized with a much lower Si/Al ratios.

One of the most interesting features of FER is the presence of the so-called FER "cavities" at the intersection of the 10- and 8-ring channels, which are only accessible through the 8-ring window (see FER cavity in Figure 2a). Thus, the control of the aluminum distribution within the FER structure should determine the acid site accessibility and, consequently, the catalytic activity/selectivity. ${ }^{22}$ Perez-Pariente et al. have rationalized the use of cooperative structure directing agents to control the aluminum distribution within the FER zeolite (see Figure $2 b$ ). ${ }^{18 d, 23}$ These authors prepared different ferrierite samples containing similar Si/Al ratios but using different combinations of organic molecules as OSDAs, such as tetramethylammonium (TMA) and pyrrolidine (PYR), TMA and 1-benzyl-1-methylpyrrolidium (BMP), and finally, only PYR molecules. Following this strategy, it has been observed that the number of acid sites in the FER cages or in the 10-ring channels varies depending on the combination of OSDAs used, and a correlation between the acid sites accessibility and catalytic activity for 1-butene isomerization has been observed. ${ }^{23}$ Moreover, the preferential population of the active sites in the 8-rings using these OSDA mixtures has allowed to increase the activity of FER zeolite for the very specific carbonylation reaction of dimethyl ether (DME) with carbon monoxide. ${ }^{24}$ Nevertheless, since mordenite zeolite (MOR, 12x8-ring pores) performs better than FER for the carbonylation of DME, the 
benefits of multipore zeolites with confined pockets for this industrially relevant reaction will be discussed later.

The most remarkable application of FER zeolite is as catalyst for the skeletal isomerization of 1-butene into isobutene. ${ }^{25}$ Isobutene is of interest because it is used to produce methyl-tert-butyl ether (MTBE), an important gasoline additive used as octane enhancer. ${ }^{26}$ MTBE, although restricted at least partially in 25 states of the US due to environmental issues, ${ }^{27}$ is still broadly employed in Europe and Asia. FER is much more effective for 1-butene isomerization than other zeolites, giving isobutene yields close to the thermodynamic equilibrium, and it is used as industrial catalyst. ${ }^{25,28}$ It has been reported that the intersected pore system of FER is very specific for the skeletal isomerization of butenes, being responsible of its higher catalytic activity. ${ }^{29}$ Nevertheless, the highest isobutene selectivities are observed after the formation of carbonaceous deposits during the initial reaction stages. ${ }^{29}$

In addition to the isomerization of 1-butene, other interesting descriptions using FER as catalyst are related to the production of light olefins (ethylene, propylene and butene) by cracking of naphtha or naphtha-related compounds. ${ }^{30}$ FER shows higher selectivities towards the desired olefins than other zeolites (i.e. ZSM-5), but much lower catalytic activities by hindered diffusion pathways, probably by the presence of the small pores. However, in a recent publication, ${ }^{30 f}$ it is shown that combining FER and ZSM-5 results in a maximized olefin yield, with FER avoiding excessive hydrogen transfer reactions, and ZSM-5 compensating for the lower activity of FER.

\section{2.- Medium-small pore zeolites synthesized using linear dicationic OSDAs}

Different multipore zeolites with intersecting medium and small pores have been synthesized using linear dicationic OSDAs (see Figure 3).

ZSM-57 (MFS topology) is composed by a two-dimensional pore system consisting of 10-ring $(5.1 \times 5.4 \AA$ A $)$ pores in the [100] direction intersecting with 8-ring (3.3 x $4.8 \AA$ ) pores in the [010] direction. ${ }^{31}$ As compared to FER, ZSM-57 presents similar 8-ring, but larger 10-ring pores $(4.2 \times 5.4 \AA)$. ZSM-57 is synthesized using the linear dicationic $N, N, N, N^{\prime}, N^{\prime}, N^{\prime}$-hexaethylpentanediammonium as OSDA (see Figure 3 ). ${ }^{32}$ Interestingly, molecular modeling studies indicate that these OSDA molecules are placed along the 10-ring channels with their positive charges in the channel intersection/lobes, directing 
consequently to a preferential positioning of the acid sites in these intersection/lobes. ${ }^{33}$ This is very interesting because the unique lobate-pore structure of ZSM-57 and the adequate acid site distribution favor the activity and selectivity for alkene oligomerizations thanks to the crosswise arrangement of alkenes. ${ }^{33}$ ZSM-57 gives higher conversions than other zeolites for oligomerization of 1-butene, with better yields to desired branched $\mathrm{C} 8$ products. ${ }^{33}$

TNU-10 (STI topology) is also composed by 10-ring channels $(4.7 \times 5.0 \AA$ ) interconnected through 8-ring channels $(2.7 \times 5.6 \AA) .{ }^{34}$ The first syntheses of this material was performed in absence of OSDA, yielding zeolites with very low Si/AI ratios (3.3-3.6). ${ }^{35}$ Recently, the use of the divalent linear 1,4-bis(N-methylpyrrolidinium)butane cation as OSDA (see Figure 3) has allowed the synthesis of the TNU-10 material with a Si/Al ratio close to $7 .^{34 b}$ The catalytic properties of TNU-10 have been evaluated for the skeletal isomerization of 1-butene due to its structural similarities as compared to FER zeolite, which is the preferred catalyst for this reaction. However, as it can be seen in Figure 4, TNU-10 gives lower isobutene selectivity than FER. Hong et al. hypothesized that the reason for this lower isobutene selectivity could be the shape of the 10-ring pores, which are more elliptical for FER (4.2 $\mathrm{\AA} \times 5.4 \AA$ ) than for TNU-10 (4.7 $x 5.0 \AA \AA$ ). If this is so, 1-butene molecules can find larger intracrystalline space within TNU-10 pores, increasing the presence of side reactions, such as dimerization, and reducing the selectivity towards the desired isobutene. ${ }^{26 b}$ Very recently, Zones et al. have reported the synthesis of the SSZ-75 zeolite, which also possesses the STI framework, but this material has been prepared under high silica conditions ( $\mathrm{Si} / \mathrm{Al}>$ 25). ${ }^{36}$ For its synthesis, Zones et al. have also used 1,4-bis(N-methylpyrrolidinium)butane as OSDA, but the synthesis was performed in the presence of fluoride anions.

ITQ-13 (ITH topology) is another molecular sieve presenting a multipore structure, which is formed by interconnected $10 \times 10 \times 9$-rings with pore diameters of $4.8 \AA \times 5.7 \AA$, $4.7 \AA$ x $5.1 \AA$, and $4.0 \AA$ x $4.9 \AA$, respectively. ${ }^{37}$ ITQ-13 is synthesized using the linear dicationic hexane-1,6-bis-trimethylammonium as OSDA (see Figure 3). ${ }^{38}$ The catalytic activity of this material has been evaluated as an additive for increasing the propylene selectivity during cracking of a vacuum gasoil. ${ }^{39}$ When compared to the conventionally used ZSM-5, the presence of interconnected medium and small channels with pore dimensions slightly smaller than those of ZSM-5, allows improving the shape-selectivity 
for cracking linear olefins in the gasoline range, and increases the propylene/propane ratio compared to ZSM-5 from 2.5 to 4 at similar conversions. ${ }^{39}$

Finally, the use of molecular modeling has allowed predicting the most favorable OSDAs to direct the crystallization of the new multipore ITQ-34 zeolite (ITR topology). ${ }^{40}$ ITQ-34 is a polymorph of ITQ-13 zeolite containing a different zeolitic sheet stacking, and it also presents an interconnected pore system of $10 \times 10 \times 9$-rings with pore diameters of $6.03 \AA$ × $4.87 \AA$, $5.25 \AA$ x $4.73 \AA$, and $4.86 \AA$ x $4.12 \AA$, respectively. As shown in Figure 3, the preferred OSDA for the synthesis of the ITQ-34 zeolite is the dicationic propane-1,3-bis(trimethylphosphonium). Up to now, a catalytic application for this material has not been reported, but attending to its multipore framework topology, it could be a useful additive for catalytic cracking in order to improve the propylene yields.

\section{3.- Medium-small pore zeolites from layered precursors}

There are several examples in the literature where layered silicates have been used as precursors for the synthesis of crystalline zeolitic frameworks, such as MWW, ${ }^{41} \mathrm{EU}$ $20 \mathrm{~B},{ }^{42} \mathrm{CDS}-1,{ }^{43} \mathrm{Nu}-6,{ }^{44}$ and RWR. ${ }^{45}$

Following the topotactic transformation, Gies et al. have reported the preparation of the multipore RUB-41 zeolite (framework type code RRO) after calcining the layered RUB-39 silicate precursor. ${ }^{46}$ This layered precursor is first synthesized under hydrothermal conditions using dimethyldipropylammonium (DMDPA) as OSDA at $150^{\circ} \mathrm{C}$, and later transformed to the novel crystalline zeolite RUB-41 by calcination in air at $600{ }^{\circ} C^{46}$ RUB-41 possesses a two-dimensional channel system with intersecting 8- and 10-ring pores of $5.0 \AA$ x $2.7 \AA$ and $6.5 \AA$ x $4.0 \AA$, respectively. ${ }^{46-47}$ This multipore molecular sieve was first synthesized as a pure-silica polymorph, ${ }^{46-47}$ being successfully applied for the selective separation of trans-2-butene and cis-2-butene from 1butene. ${ }^{46}$ However, for its application as an acid catalyst, the incorporation of Al atoms in the framework of RUB-41 is required. The direct introduction of Al atoms in the RUB-39 synthesis gel mixture, resulted in a limited Al incorporation in the RUB-41 structure after calcination (Si/Al 120). ${ }^{48}$ To improve the Al incorporation, Yilmaz et al. developed a two-step synthesis procedure. ${ }^{48}$ In the first step, a synthesis gel containing the silica precursor, the organic DMDPA molecules acting as OSDAs, and 3-5\%wt seeds 
of RUB-39 material, was autoclaved at $150^{\circ} \mathrm{C}$ for 48 hours. In a second step, the aluminum precursor was added to the above synthesis gel, and introduced in the oven at $150^{\circ} \mathrm{C}$ for 48 hours. ${ }^{48}$ Following this two-step procedure, the Si/Al ratio in the final solid could be decreased to 20 , being most of these Al atoms in tetrahedral coordination. $^{48-49}$

The catalytic activity of Al-RUB-41 has been studied for the methanol amination reaction. $^{48}$ Methylamines are important intermediates in the synthesis of pharmaceuticals, pesticides or surfactants. The typical products in the methanol amination reactions are monomethylamine (MMA), dimethylamine (DMA), and trimethylamine (TMA). The most demanded products are MMA and DMA but,

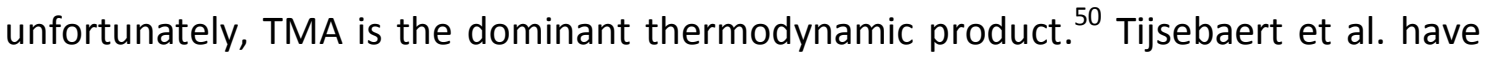
shown that AI-RUB-41 notoriously enhances the production of the desired MMA and DMA products as compared to other shape-selective catalysts. ${ }^{49}$ The benefits of the shape-selective structure of Al-RUB-41 are maximized at low Al contents (Si/Al 160), probably due to the reduction of unselective external acid sites.

Very recently, the synthesis of the new multipore IPC-4 zeolite (framework type code PCR) has been described by Russell et al. using the germanosilicate UTL zeolite as precursor. ${ }^{51}$ This germanosilicate can be considered as high silica layered material, where these high-silica layers are interconnected by double-4-rings (D4R) units that are preferentially occupied by Ge atoms. The specific location of the Ge atoms in the interlayer region allows the formation of a high-silica layered intermediate by the selective removal of these Ge atoms via hydrolysis under slightly acidic conditions. The final calcination of this layered intermediate results in the crystalline IPC-4 zeolite, which possesses a two-dimensional channel system with intersecting 8- and 10-ring pores of $4.7 \AA$ x $3.5 \AA$ and $6.1 \AA$ x $5.1 \AA$, respectively. ${ }^{51}$

\section{3.- Zeolites with small and large pores}

Numerous zeolites presenting interconnected large and small channels have also been described in the literature (see Table 1). Unfortunately, the chemical compositions required for the preparation of most of these multipore materials are limited to AIPOrelated or to zeolites with low-silica frameworks, yielding materials with low hydrothermal stability in most cases. The paradigmatic zeolite containing 
interconnected large and small pores is mordenite (MOR), which can be prepared under large synthesis conditions and, very interestingly, its chemical composition and crystal size can be controlled. Consequently, a considerable number of catalytic applications have been reported for MOR, which will be covered in section 3.3. However, we will first summarize the syntheses and applications of other relevant molecular sieves with interconnected 12x8-ring channels.

\section{1.- AIPO-related materials presenting interconnected 12x8-ring pores}

Different multipore AIPO-related zeotypes with interconnected 12x8-ring pores have been described. SAPO-40 is a silicoaluminophosphate with AFR topology (see Figure 5), which consists of a two dimensional channel system with a 12-ring pore ( $6.7 \AA$ ) along the $c$-axis interconnected with an 8-ring pore ( $3.8 \AA$ ) along the $b$-axis. ${ }^{52}$ SAPO-40 can be prepared using tetrapropylammonium hydroxide as OSDA, and the acidity of this material can be modulated by controlling the amount and distribution of silicon atoms in the framework. ${ }^{53}$ The acidity of SAPO-40 has been evaluated for $m$-xylene isomerization/disproportionation ${ }^{54}$ and for the ethylbenzene disproportionation. ${ }^{55}$ This material shows lower initial selectivity for isomerization than for disproportionation (I/D ratio) in the $m$-xylene isomerization reaction, indicating that the available void volume in SAPO-40 favors the formation of the bulkier transition state required for $\mathrm{m}$ xylene disproportionation. ${ }^{54}$ On the other hand, the distribution of the diethylbenzene isomers for the ethylbenzene disproportionation obtained when using SAPO-40 as catalyst is close to the thermodynamic equilibrium, revealing the absence of shapeselectivity. ${ }^{55}$ The high void volume of the structure of SAPO-40 due to the presence of regular side pockets or lobes could explain these catalytic results.

Recently, researchers at Chevron and Exxon have reported a new AlPO-based material with the SFO framework topology (named SSZ-51 ${ }^{56}$ and EMM- $8,{ }^{57}$ respectively), which is closely related to the structure of SAPO-40. SFO has been synthesized in its aluminophosphate and silicoalumiophosphate forms in the presence of 4dimethylaminopyridine as OSDA. ${ }^{56-57}$ The SFO structure consists of a two-dimensional intersecting channel system, where one of the channels is defined by an 8-ring window along the $b$-axis, and the other channel is defined by a 12-ring window along the $c$-axis. The pore topology is essentially the same as the one observed in SAPO-40, but a 2-fold 
rotation around the $a$ axis introduces the crystallographic differences between these two highly-related frameworks (see Figure 5).

Other metalloaluminophosphates (MeAIPOs) containing interconnected large and medium pores have also been described, such as magnesioaluminophosphate MAPO-

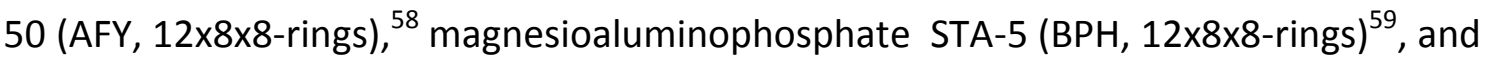
Co-, Mn-, Co- aluminophosphates UCSB-6, UCSB-8, and UCSB-10 (SBS, 12x12x8-rings; SBE, 12x8-rings; and SBT, $12 \times 12 \times 8$, respectively). ${ }^{60}$ Catalytic applications for these MeAIPOs have not been reported, probably due to the limited hydrothermal stability of these metal-containing materials. ${ }^{58}$

\section{2.- Low silica zeolites containing interconnected $12 \times 8$-ring pores}

Different zeolites with intersected 12- and 8- pores, such as UZM-4 (BPH, 12x8x8-rings,

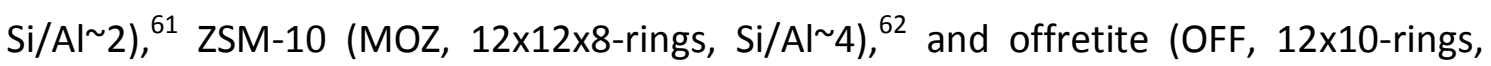
$\left.\mathrm{Si} / \mathrm{Al}^{\sim} 3-8\right),{ }^{63}$ have been reported in the literature presenting low $\mathrm{Si} / \mathrm{Al}$ ratios.

The first silicoaluminate synthesized with BPH structure was zeolite $Q$, which was prepared in the presence of different inorganic cations leading to Si/Al ratios of $1 .^{64}$ The Si/Al ratio could be increased to almost 2 by using a mixture of $\mathrm{Li}^{+}, \mathrm{TMA}^{+}$, and TEA as templates in the case of UZM- 4 zeolite, ${ }^{61}$ and to almost 6 by combining the above template mixture and the presence of pre-formed UZM- 4 crystals as seeds. ${ }^{65} \mathrm{BPH}$ topology shows a three-dimensional pore system with a straight 12-ring channel

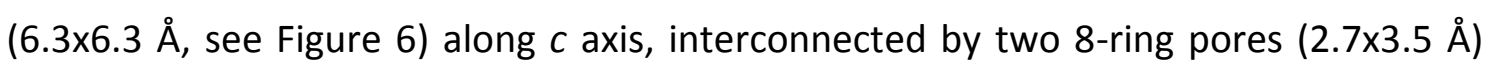
along the plane $a b$.

ZSM-10 zeolite, showing the MOZ topology, was synthesized with potassium and 1,4dimethyl-DABCO cations as OSDA, presenting a Si/Al ratio in the final solid of almost $4{ }^{62,66} \mathrm{MOZ}$ topology shows two different one-dimensional 12-ring channels along the $c$ axis (see Figure 6), with one of these channels being identical to the 12-ring sinusoidal channel of the LTL zeolite $(6.8 \times 7.0 \AA)$, and the other to the 12-ring channel of OFF

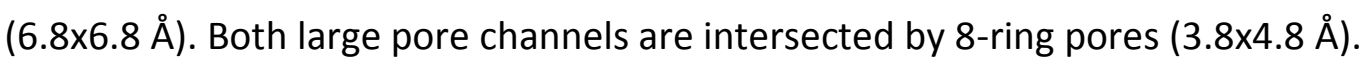

Finally, the early syntheses of pure OFF zeolite were reported using sodium and potassium cations as inorganic SDA, resulting in very low silica materials (Si/AI 3-5). ${ }^{67}$ However, Sano et al. have recently increased the Si/Al ratio to almost 8 in the final OFF 
zeolite, using dealuminated FAU zeolites as silicoaluminate precursors in the presence of benzyltrimethylammonium, lithium and sodium cations. ${ }^{63}$

OFF topology shows a two-dimensional pore system of 12 -ring channels $(6.7 \times 6.8 \AA$, see Figure 6) along the $c$ axis, interconnected by 8-ring channels (3.6x4.9 $\AA$ ) along the $a$ axis. Catalytic results have been described for the offretite, such as $m$-xylene isomerization, ${ }^{68}$ and more recently, selective catalytic reduction of NOx with ammonia on the $\mathrm{Cu}(\mathrm{II})$-ion exchanged zeolite. ${ }^{69}$ While other potential applications of OFF have also been described for oil dewaxing ${ }^{70}$ or naphthalene cracking. ${ }^{71}$ However, its use is strongly limited by the low resistance to coking and high catalyst deactivation rates. ${ }^{72}$ In general, the catalytic applications of low-silica materials are restricted by their low acidity and low hydrothermal stability. In this sense, synthesis procedures directed to increase the Si/Al ratio of the above materials can result in zeolite samples that can go to further post-synthesis treatments. This will open the possibilities of those zeolites for different catalytic processes.

\section{3- Mordenite}

The framework of MOR shows parallel 12-ring pores $(6.5 \times 7.0 \AA ̊)$ and 8-ring pores (2.6 x $5.7 \AA$ ) along $c$-axis, interconnected by 8 -ring pores $(3.4 \times 4.8 \AA$ ) along $b$-axis (see Figure 7). ${ }^{73}$ This material can be synthesized under a broad range of synthesis conditions, allowing the control of the framework Si/Al ratio (4 to 25 ) by direct synthesis, ${ }^{74}$ the crystal sizes (from $20 \mathrm{~nm}$ to $160 \mu \mathrm{m}$ ), ${ }^{75}$ crystal morphologies (needles, flat crystallites, acicular crystals, prismatic crystals, among others), ${ }^{75 a, 76}$ and even the aluminum distributions within the MOR crystals. ${ }^{77}$ Due to this large synthetic spectrum, MOR has become one of the most important industrial catalysts with application in different chemical processes, such as alkylation, dewaxing, reforming, cracking or hydroisomerization reactions. ${ }^{75 c, 78}$ For instance, a highly thio-resistant metal-based MOR catalyst is being applied commercially for light naphtha isomerization ${ }^{2 c, 79}$ to increase the octane number. However, despite the multipore nature of MOR (interconnected large and small pores), this zeolite is considered as a one-dimensional large pore framework when used as catalyst for most of the above described industrial applications due to the small size of the 8-ring pores, ${ }^{75 c}$ and very 
few examples can be found where the 8-ring pores are considered to play a predominant role.

One case that clearly shows the relevance of the presence of the 8-ring pores is the high rate of coke oxidation observed for MOR, comparable to that of the threedimensional HY. This was attributed to the diffusion of oxygen not only through the 12ring pores, which are blocked by the carbonaceous compounds, but also through the 8-ring channels. ${ }^{80}$

There is another case in where the presence of the 8-ring pores in the mordenite has been shown to be key for the catalyst. This is the selective carbonylation of methanol and dimethyleter (DME) with $\mathrm{CO}$ to acetic acid. ${ }^{16 c, 17 a, 81}$ Acetic acid is industrially produced using organometallic complexes as catalysts and iodide compounds as promoters. ${ }^{82}$ Then, the replacement of the homogeneous by a solid catalyst able to catalyze the carbonylation reaction in absence of iodides would be industrially relevant. Researchers at BP first described that $\mathrm{CU}-\mathrm{MOR}$ catalysts were active and selective for the carbonylation of methanol in the absence of any halide promoter. ${ }^{81 a, b}$ Then, Iglesia et al. found that the carbon-carbon formation via $\mathrm{CO}$ insertion into chemisorbed methyl groups occurs selectively within 8-ring pores of some acid zeolites, and MOR is particularly active compared to other 8-ring pore zeolites (i.e. FER). ${ }^{17 a}$ Indeed, DME carbonylation rates are proportional to the number of acid sites within the 8-ring pores. ${ }^{17 a}$

If one looks the structure of MOR, it is possible to see the presence of side pockets in the intersection of 12 - and 8-ring pores (see Figure 7B). Moreover, there are four nonequivalent tetrahedral sites in the unit cell of MOR (T1 in the 12-ring channel, T2 and T4 in the intersection between the 12-ring channel and 8-ring pocket, and T3 inside the 8-ring pocket). ${ }^{16 c}$ Theoretical work shows that methanol carbonylation only occurs selectively in the T3 site, which is placed inside the 8-ring pockets (see Figure 7C). ${ }^{16 c, 17 c}$ This high selectivity can be explained by the unusual orientation of the chemisorbed methoxy group in this confined site, allowing a good fitting between the linear transition state and the stabilizing framework oxygens. In all other positions, undesired reactions (such as formation of DME or hydrocarbons) compete with the carbonylation reaction, driving into the catalyst deactivation. 
Beyond the benefits of the multipore topologies, the carbonylation of methanol catalyzed by MOR is a particularly interesting case of site specificity upon confinement that was not described previously in the literature. This example reveals the remarkable impact that the rationalization on zeolite synthesis can have on shapeselective catalysis, not only in terms of achieving new pore topologies, but also for introducing the framework Al, and therefore the active sites, in particular framework positions.

\section{4.- Zeolites with medium and large pores}

The first zeolite described presenting interconnected large and medium pores in the same structure was the natural occurring boggsite mineral. ${ }^{83}$ This discovery revealed the possibility of preparing structures with intersected large and medium channels, which should allow preferential diffusion pathways for reactants and products of different sizes, as compared to zeolites with exclusively large or medium pores. Recently, boggsite has been synthesized in the laboratory within a larger framework $\mathrm{Si} / \mathrm{Al}$ composition and it is named ITQ-47. We will show below that multipore zeolites with medium and large pores, show exceptional catalytic activities not previously reported when used as shape-selective catalyst. ${ }^{84}$

\section{1- CON family (SSZ-26, SSZ-33, CIT-1, and ITQ-24)}

SSZ-26 and SSZ-33 zeolites were the first synthetic zeolites reported in the literature containing interconnected 12 - and 10 -ring pores. ${ }^{85}$ Unfortunately, as it will be shown later, these materials perform as large cages connected by 10- and 12-ring windows rather than catalysts with well-defined intersecting 10- and 12-ring channels. These zeolites are members of a family of crystalline materials, known as the CON family, where initially two different polymorphs with different stacking layers were proposed ( $A B A B$...sequence and $A B C A B C$...sequence for polymorphs $A$ and $B$, respectively, see Figure 8). ${ }^{86}$ In the case of SSZ-26 and SSZ-33, both materials are formed by the intergrowth of the two proposed polymorphs, containing A/B polymorph enrichments of $15 / 85$ and $30 / 70$, respectively. ${ }^{86}$ SSZ-26 and SSZ-33 are prepared as aluminosilicate and borosilicate forms, respectively, using similar polycyclic ammonium OSDAs (see Figures $9 \mathrm{a}$ and $9 \mathrm{~b}) .{ }^{86}$ It has been reported that, at least in the case of SSZ-26, there is a 
clear relationship between the organic structure-directing agent and the pore geometry (see Figure 9c). ${ }^{86}$ Besides SSZ-26 and SSZ-33, Davis et al. were able to synthesize the pure crystalline polymorph B form of the CON-family (see Figure 8), which was named CIT-1 zeolite. ${ }^{87}$ This material was synthesized in its borosilicate form using $N, N, N$-trimethyl-cis-myrtanylammonium as OSDA. ${ }^{87}$

In addition to the polymorphs $\mathrm{A}$ and $\mathrm{B}$ of the CON family, a third polymorph (named polymorph C, see Figure 8 ) was also proposed. ${ }^{86}$ This hypothetical polymorph was constructed from the stacking of polymorph A layers following an AAA...stacking sequence. ${ }^{86}$ Although Lobo et al. did not observe any experimental evidence for the presence of the polymorph $\mathrm{C}$, they suggested that its preparation may be possible by properly rationalizing the synthesis conditions. Indeed, we considered that the main structural difference between the hypothetical polymorph $\mathrm{C}$ of the CON family and the others, is that the former contains double-4-rings (D4R) as secondary building units (SBU). Then, since we demonstrated previously that Ge could direct to the synthesis of new zeolitic frameworks containing $D 4 R,{ }^{88}$ it was logical to attempt the synthesis of the polymorph $\mathrm{C}$ of $\mathrm{CON}$ in the presence of Ge. Following the Ge approach, the pure polymorph $\mathrm{C}$ of the CON family in its silicogermanate form (named ITQ-24, with a Si/Ge ratio of $\sim 5$ ), and using hexamethonium as OSDA was achieved. ${ }^{89}$ Thus, by further rationalization we were able to perform the synthesis of ITQ-24 under Ge-free conditions. ${ }^{90}$ We considered that the introduction of B instead of Ge would produce small changes on the Si-O-X angles (where $\mathrm{X}$ could be $\mathrm{Ge}$ or $\mathrm{B}$ ), and fluoride anions could stabilize the D4Rs present in ITQ-24 zeolite. Furthermore, the use of bulky and rigid bicyclic OSDAs (see for instance the OSDA shown in Figure 10b) would reduce the positive charges when filling the void volume of ITQ-24 increasing, therefore, the framework Si/Al ratio. Following the above hypothesis, the Ge-free borosilicate form of ITQ-24 was successfully achieved using medium sized dicationic hexamethonium cation as OSDA, and the pure silica ITQ-24 form was synthesized using the bulky and rigid OSDA shown in Figure 10b. ${ }^{90}$

The catalytic behavior of CIT-1/SSZ-33/SSZ-26 has been evaluated for the isomerization/disproportionation of $m$-xylene. ${ }^{91} \mathrm{CIT-1/SSZ-33/SSZ-26}$ materials reveal catalytic properties that resemble more to those of 12-ring pores rather than to 10 ring pores. ${ }^{91 a}$ In general, medium pore zeolites (i.e. EU-1 or ZSM-5 zeolites) show $p$ - 
xylene/o-xylene $(p / o)$ ratios above 2.5 while large pore zeolites give values close to 1 (i.e. Beta zeolite). ${ }^{68 b, 92}$ As seen in Figure 11, CIT-1/SSZ-33/SSZ-26 materials show $p / o$ ratios around 1.3-1.5, values which are more characteristic of large pore zeolites. On the other hand, SSZ-33 and CIT-1 show intermediate isomerization/disproportionation $(i / d)$ ratios $(\sim 7-10)$, similar to the values reported for large pore zeolites. The typical $(i / d)$ ratios obtained with medium pore zeolites are much higher (above 25) since disproportionation reactions, which require large void spaces, are sterically hindered within the medium pores. From these results, Davis et al. concluded that CIT-1/SSZ33/SSZ-26 materials behave as an ensemble of large cages connected by 10- and 12ring windows rather than as a material with well-defined intersecting 10- and 12-ring channels. ${ }^{91}$

In agreement with the above, the study of the diffusion of alkylaromatics (para- and ortho-xylenes) through this family of zeolites, ${ }^{93}$ show that that the diffusion of $o$-xylene occurs exclusively through the 12-ring channels, while $p$-xylene can diffuse through the 12- and 10-ring pores. Interestingly, molecular modelling studies reveal that, although o-xylene cannot diffuse through the 10-ring pores, it spends considerable time, according to the simulation, diffusing through the intersection of the 12- and 10-ring channels. This means that large molecules, as for instance trimethylbenzenes, will not diffuse trough the 10-ring pores but can penetrate through the openings of these 10ring channels. Taking into account all of the above, it appears that the multipore zeolites belonging to the CON family should be considered as zeolitic structures formed by large void volumes connected by 12 - and 10-ring windows, and not as multipore structure with well-defined pores of different dimensions.

\section{2- Boggsite}

Boggsite (BOG) was the first natural zeolitic material discovered containing interconnected large and medium pores. ${ }^{83}$ Unfortunately, this mineral has only been found in minor quantities in Oregon and the Antarctica. ${ }^{83,94}$ Since the discovery of this zeolite in its natural form, the possibility to synthesize this material in the laboratory became a highly challenging subject in zeolite synthesis. ${ }^{93 a}$ Very recently, the synthetic BOG structure, named as ITQ-47, has been obtained as borosilicate form by using, for the first time, phosphazene molecules as OSDAs. ${ }^{95}$ Interestingly, phosphazene derived 
molecules are very attractive OSDAs since a broad family of molecules can be easily prepared by simple substituent modifications. ${ }^{95}$ The selection of the most adequate OSDA for the BOG synthesis was previously evaluated by molecular modelling. This "insilico" analysis predicted the optimum stabilization of BOG when the OSDA shown in Figure 10c was used. Then, a complete experimental design studying the combination of this OSDA together with other relevant variables allowed the synthesis of ITQ-47 zeolite with the BOG framework topology. ${ }^{95}$ ITQ-47 structure contains a bidirectional pore system presenting 12-ring straight channels with circular openings of $7 \AA$, interconnected to 10-ring channels with pore openings of $5.5 \times 5.3 \AA$.

The catalytic activity of the Al-exchanged ITQ-47 was evaluated for liquid phase alkylation of benzene with propylene to produce cumene. Due to its structure, with interconnected 12- and 10-ring channels, ITQ-47 was expected to be not only adequate, but also more selective to the desired mono-alkylation product than the 12 ring Beta zeolite, which is commercially employed for the production of cumene. ${ }^{95}$ The catalytic results confirm this assumption, and ITQ-47 gives higher cumene selectivities and lower yields of undesired secondary products, such as n-propylbenzene, when compared to the large pore Beta zeolite.

\section{3- MCM-68}

MCM-68, a molecular sieve with MSE topology, shows a multipore system with interconnected and well-defined $12 \times 10 \times 10$-ring pores. ${ }^{96}$ The synthesis of this MCM-68 was first reported by researchers at ExxonMobil in its silicoaluminate (Si/Al ratios 912) form and using the OSDA shown in Figure $10 \mathrm{~A} .{ }^{96}$ It is important to notice that this OSDA is very similar in shape, size and form to the OSDA used for the synthesis of the pure silica ITQ-24 (see Figure10B), ${ }^{90}$ being both molecules very selective OSDAs for crystallizing multipore zeolite containing interconnected large and medium pores.

The silicoaluminate form of MCM-68 has been tested for different acid catalyzed reactions, and MCM-68 zeolite performs as an efficient catalyst for the shape selective alkylation of biphenyl ${ }^{97}$ and naphthalene. ${ }^{98}$ Indeed, very high selectivities towards 4,4'-diisopropylbiphenyl ( 80\%), 4,4'-di-sec-butylbiphenyl ( 90\%), and 4,4'-di-tertbutylbiphenyl ( 95\%), have been reported for the biphenyl isopropylation, secbutylation, and tert-butylation, respectively..$^{97-98}$ Shape-selectivity increased with 
increasing the size of the alkylating agent and, in fact, shape-selectivity for alkylating naphthalene was only noticeable for the bulkier 1-butene and isobutene. ${ }^{98}$ In both cases, partial dealumination of the zeolite to Si/Al values around 70 was seen to improve catalytic activity. These high selectivities have been explained by the restricted transition state mechanism inside the straight 12-ring channels, precluding the transition states to form bulky isomers. Lower selectivites have been observed for

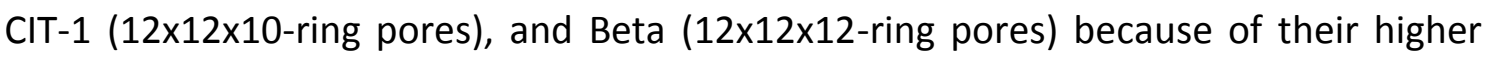
space at their channel intersections. ${ }^{99}$ This is a clear example of the different selectivities observed when comparing multipore structures with well-defined discrete pores, such as MCM-68, and others, such as CIT-1, which can be considered as a zeolite with 12-ring pores connected by 10-ring windows that present a catalytic behavior closer to that of the large pore Beta.

Partially dealuminated MCM-68 zeolite has also been shown to be an efficient catalyst for the selective formation of propylene by hexane cracking. ${ }^{100}$ Dealuminated MCM-68 (Si/Al 50) shows higher propylene selectivity ( 50\%) compared to other zeolite catalysts (MFI, MOR or Beta) within the studied reaction temperature range (450 to $\left.600^{\circ} \mathrm{C}\right),{ }^{100}$ indicating that MCM-68 could be a good candidate to be used as FCC additive for increasing the propylene production. The authors speculate that the hexane cracking must occur on the acid sites predominantly present in the welldefined 10-ring pores of the dealuminated MCM-68 zeolites. Thus, a selective dealumination of MCM-68 involving the framework Al atoms facing the 12-ring pores is suggested. Moreover, the specific multipore structure of MCM-68 with continuous $12-$ and 10- ring pores, and the selective dealumination of the large pores were also related to its improved coking tolerance as compared to the other studied zeolites.

Finally, the titanosilicate form of the MCM- 68 zeolite has also been synthesized by a simple post-synthetic isomorphous substitution of Ti for Al. ${ }^{101}$ Ti-MCM-68 has been tested for the industrially relevant phenol hydroxylation reaction using $\mathrm{H}_{2} \mathrm{O}_{2}$ as oxidant, resulting in higher para-selectivity and higher $\mathrm{H}_{2} \mathrm{O}_{2}$ efficiency, compared to industrially useful TS-1. ${ }^{101}$ The high activity was related to the higher diffusivity through 12-ring versus 10-ring channels, whereas the high para-selectivity was ascribed to the absence of large inner cavities. 


\section{4.- Zeolites of the SSZ family containing large and medium pores (SSZ-56, SSZ-57, and SSZ-82)}

In the last years, researchers at Chevron have reported three new zeolites presenting large and medium pores in the same structure, i. e. SSZ-56, SSZ-57, and SSZ-82. SSZ-56 (SFS) is a borosilicate with a two-dimensional channel system of intersecting 10-ring pores with openings of $5.5 \times 4.8 \AA$, and 12-ring pores with elliptical openings of $8.4 \mathrm{x}$ $5.9 \AA$ (see Figure 12A). ${ }^{102}$ The most interesting feature of the SSZ-56 synthesis is the extremely high isomer specificity required for the OSDAs used in its preparation. SSZ56 synthesis strictly requires the presence of the trans-fused isomer of $\mathrm{N}, \mathrm{N}$-diethyl-2methyldecahydroquinolinium as OSDA, because the presence of the cis-fused isomer results in the formation of layered or dense phases. ${ }^{102}$

SSZ-82 (SEW) shows a two-dimensional interconnected 10-/12-ring channel system, where the 10-ring pores form a zig-zag channel with openings of $4.9 \times 5.5 \AA$ along the $a$ axis. These 10-ring pores intersect with the straight 12-ring pores with elliptical openings of $5.2 \times 8 \AA$ along the $b$ axis (see Figure 12B). ${ }^{103}$ This multipore zeolite can be synthesized as borosilicate form using 1,6-bis( $N$-cyclohexylpyrrolidinium)hexane dication as OSDA. ${ }^{104}$

Recently, Zones et al. have reported the synthesis of the SSZ-57 (SFV topology). ${ }^{105}$ This material presents large domains of intersecting 10-ring pores, which are interrupted every $110 \AA$ by isolated and well-defined large pores (the 12-ring:10-ring ratio is 1:15, see Figure 12C). This is one of the most complex zeolitic structures solved, with $99 \mathrm{Si}$ atoms in the asymmetric unit, and advanced crystallographic techniques (structure solution in four-dimensional space and interpretation of three-dimensional diffuse scattering by Monte Carlo simulation) were required for its structure solution. ${ }^{105}$ SSZ57 can be directly synthesized in its aluminosilicate and borosilicate forms. The direct synthesis of the aluminosilicate SSZ-57 results in an acid material that is catalytically active for some model reaction, such as the conversion of methanol to hydrocarbons, xylene isomerization, or catalytic cracking. ${ }^{106}$ In contrast, the borosilicate form of SSZ57 shows an acid strength too weak to be active in hydrocarbon conversion reactions. Nevertheless, an isomorphic substitution of B with Al atoms can be performed under acid conditions. ${ }^{107}$ Interestingly, this post-synthetic isomorphic heteroatom substitution only occurs in the large pores. Thus, following this procedure, it has been 
possible to prepare the SSZ-57LP material, in where the strong acid sites are only found within the large pores of the SSZ-57 zeolite. ${ }^{108}$ This preferentially distribution of acid sites results in significant differences regarding catalytic selectivity, as compared to regular Al-SSZ-57, with a catalytic behavior closer to pure large-pore zeolites. ${ }^{109}$

\section{5.- ITQ-22}

ITQ-22 (IWW) is a zeolite that presents interconnected 8-, 10- and 12-ring channels with pore apertures of $4.52 \times 3.32,5.86 \times 4.98$, and $6.66 \times 6.66 \AA$, respectively, within the same structure. ${ }^{110}$ The channel system of this zeolite is three-dimensional with the 12- and 8-ring pores running along the $c$ axis, which are intersected by a sinusoidal 10ring pore running normal to the $c$ axis (see Figure 13). This unique zeolite has been synthesized by combining the organic molecule 1,5-bis-(methylpyrrolidium)-pentane as OSDA, and the structure directing effect of Ge towards D4Rs. ${ }^{110}$

The catalytic activity of ITQ-22 has been first evaluated for the $m$-xylene isomerization and disproportionation. ${ }^{110}$ It shows an intermediate catalytic behavior for the $m$-xylene isomerization as compared to zeolites presenting exclusively 10 - or 12 -ring pores ( $p / o$ ratios of 1.2, 2.3, and 1.8 for Beta, ZSM-5, and ITQ-22, respectively). ${ }^{110} \mathrm{~A}$ similar intermediate catalytic behavior is also observed when analyzing the isomerization/disproportionation $(i / d)$ ratios $(2.7,34.3$, and 9.1 for Beta, ZSM-5, and ITQ-22, respectively). Moreover, a significant difference can be observed between the $\mathrm{i} / \mathrm{d}$ ratio for ITQ-22 and SSZ-33, which is another multipore zeolite presenting interconnected medium and large pores (the $i / d$ ratios are 1.8 and 9.1 for SSZ-33 and ITQ-22, respectively). SSZ-33 performs similarly to large pore zeolites, and the reason for this is that the structure of SSZ-33 can be considered as a large pore zeolite with 10-ring windows connecting adjacent 12-ring pores. In contrast, ITQ-22 shows a real intermediate behavior because of the steric hindrance imposed by the 10-ring pores to the formation of bulky transition state complexes, revealing that in the case of ITQ-22 there are continuous 10-ring channels communicating between two neighboring 12ring channels. ${ }^{110}$ In addition, ITQ-22 is a very active and selective catalyst, not only for the synthesis of ethylbenzene and cumene, by alkylating benzene with ethanol and 2propanol, respectively, in vapor phase (see Figure 14), but also for the liquid phase

production of cumene by alkylation of benzene with propene under reaction 
conditions relevant to the industrial process. ${ }^{15 f}$ The higher selectivity to cumene as compared to ZSM-5 and Beta zeolites can be explained by two reasons: on one hand, the multipore nature of the ITQ-22 zeolite, which enhances the diffusion of primary products before being involved in undesired consecutive reactions and, on the other hand, to the preferential location of the acid sites in the intersections of the 10- and 12-ring pores, as revealed by theoretical calculations. ${ }^{15 f}$ It is important to note that the ITQ-22, despite containing Ge, remains stable after calcination at $5800^{\circ} \mathrm{C}^{15 f}$

Very interestingly, two different groups have recently described the preparation of highly degermanated ITQ-22 zeolites by post-synthetic acid treatments, followed by the incorporation of Si and/or Al species at created vacant sites. ${ }^{111}$ These procedures allow the synthesis of well-structured high-silica ITQ-22 zeolites with good acid properties, and improved hydrothermal stabilities.

\section{6- ITQ-39/ITQ-38}

Very recently, the selection of a type of OSDAs for the specific synthesis of multipore zeolites containing interconnected large and medium pores has been rationalized. ${ }^{112}$ To do this, the authors designed an OSDA that combines rigidity (introduced by a piperidine-derivative molecule), and certain flexibility (introduced by flexible propyl/ethyl chains, see Figure 15A). ${ }^{112}$ The selection of this structure was based on the fact that the rigid piperidine-derivative OSDAs were able to direct the crystallization of large pore zeolites (such as ZSM-12, see Figure 15A) while flexible OSDAs containing propyl chains were effective OSDAs for medium pore zeolites (such as ZSM-5, see Figure 15A). Thus, by using the OSDA with combined rigid and flexible molecular components (see Figure 15A), the synthesis was directed toward the crystallization of the new ITQ-39 zeolite containing interconnected large and medium pores in the same structure (see Figure 15B)..$^{84,113}$ ITQ-39 is formed by the intergrowth of three related polymorphs, all of them containing straight pairwise 12-ring pores along $b$ direction, interconnected by zig-zag 10-ring channels along $a$ and $c$ directions (see Figure 15B). This zeolitic structure is the most complex ever solved due to the presence of three different polymorphs containing stacking faults and twinning with nano-sized domains. ${ }^{84}$ 
ITQ-39 can be prepared under a broad range of Si/Al ratios (from 10 to pure silica), making this material very attractive regarding its potential application for industrially relevant chemical processes. Indeed, ITQ-39 has been applied as a good catalyst for the conversion of low value naphtha fractions into diesel. ${ }^{84}$ This process involves the alkylation of aromatics with olefins present in the same or in two different low valued naphtha streams, to produce monoalkylated products in the diesel range. As seen in Figure 16, ITQ-39 performs better than commercially available Beta and MWW materials, giving higher conversions, better yields and longer catalyst lifetimes. ${ }^{84}$ Although Beta shows a higher initial activity, it deactivates fast and yields lower diesel when increasing time on stream (see Figure 16). The excellent catalytic behavior of ITQ-39 for naphtha alkylation can be explained by its unique multipore topology. The straight 12-ring channels facilitate the formation and diffusion of alkylated products, while the 10-ring pores avoid the olefin oligomerization, and thus, the coke formation. The titanosilicate form of the ITQ-39 was also prepared, leading to the first direct synthesis description of a multipore metallosilicate containing interconnected large and medium pores. ${ }^{114}$ Preliminary results have shown that Ti-ITQ-39 is very active for the selective oxidation of olefins with $\mathrm{H}_{2} \mathrm{O}_{2}$.

In addition to ITQ-39 zeolite, the synthesis of a new multipore zeolite containing interconnected large and medium pores, ITQ-38 has been reported. ${ }^{115}$ This zeolite can be prepared by the combination of a dicationic piperidine-derivative OSDA with germanium atoms. ITQ-38 structure is formed by a three-dimensional framework comprising straight 10- and 12-ring channels along $b$ direction, and zig-zag 10-ring channels along $a$ and $c{ }^{115}$

\section{7.- Zeolites with interconnected large and medium pores synthesized from layered precursors}

FER, a multipore zeolite presenting a structure with interconnected small and medium pores, has already been described in section 2.1. However, FER-related materials are also known to be precursors for the synthesis of other multipore zeolites. Indeed, it has been described that the crystalline layered precursor of FER, named PREFER, ${ }^{116}$ can be converted into a new open multipore structure, APZ-4, by pillaring the interlayer region with $\mathrm{Si}$ atoms (see Figure 17a). ${ }^{117}$ In this case, the formed multipore APZ-4 
zeolite shows interconnected $12 \times 10$-ring pores, where the structure is formed by $\mathrm{SiO}_{2}(-$ $\mathrm{OH})_{2}$ bridges between terminal oxygen atoms of neighboring silicate layers (see Figure 17a). To obtain this multipore structure, the original PREFER material is treated following an acid treatment, allowing the migration of some Si fragments into the interlayer space. APZ-4 shows high hydrophilicity due to the presence of a large number of hydroxyl groups in the interlayer region. As far as we know, this material has only been synthesized in the pure silica form, and the synthesis of other APZ-4 polymorphs, such as the aluminosilicate and the titanosilicate forms, would be very interesting to evaluate their catalytic properties in different chemical processes.

The preparation of other multipore zeolite presenting large and medium pores in its structure has been recently described trough the inverse sigma transformation. ${ }^{118}$ This concept is based on the transformation of a known zeolite into a new structure by framework contraction through selective removal of a layer of framework atoms. In this sense, Verheyen et al. proposed the transformation of the silicogermanate UTL, ${ }^{119}$ which was the first multipore extra-large pore zeolite described containing 14x12-ring pores (as it will be described later) into a new multipore zeolite presenting interconnected large and medium pores. The authors claimed that the preferential location of germanium atoms in the double-4-rings (D4R) units present in the UTL structure (see Figure 17b-left), combined with their high reactivity in strong acid conditions, favor the selective removal of Ge in D4R from the UTL framework resulting in a new zeolite (see Figure 17b). Indeed, this methodology has allowed the synthesis of the new high-silica zeolite COK-14 (OKO), which does not only presents a new multipore $12 \times 10$-pore topology, but also shows a good hydrothermal stability. ${ }^{118}$ One of those zeolites, IPC-2, also presents the OKO pore topology. Both COK-14 and IPC-2 zeolites have been prepared as almost pure silica polymorphs, and thus, the preparation of the aluminosilicate form of these materials is a matter of interest to evaluate their catalytic properties.

\section{5.- Zeolites with extra-large pores interconnected with smaller pores}

In the last years, various extra-large multipore zeolites have been reported in the literature. $^{112,120}$ If the chemical composition of these extra-large pore zeolites is considered, it can be observed that most of them contain large amount of Ge atoms in 
their framework. ${ }^{112,120}$ Several years ago, Corma et al. proposed the introduction of Ge atoms in the synthesis of zeolites as inorganic structure directing agents to favor the crystallization of molecular sieves with D4R with the objective of preparing zeolite structures with low framework densities (FD, number of tetrahedral atoms per 1000 $\AA^{3}$ ) and, hopefully, with extra-large pores. ${ }^{121}$ This hypothesis was based on the theoretical prediction made by Brunner and Meier for pure silica zeolites, where a relationship was suggested between zeolites with low FD and the presence of 3- and 4rings in their structures. ${ }^{122}$ Since the crystallization of pure silica zeolites containing these small rings was difficult, the authors considered that the introduction of Ge will preserve the framework electroneutrality while changing in the wright way the T-O-T angles, facilitating the formation and stabilization of these small rings (as for instance double-3-rings, D3R, and double-4-rings, D4Rs).,120 This initial hypothesis was validated both theoretically and experimentally ${ }^{121}$ and, since then, a large amount of silicogermanates with low FD, and particularly, with extra-large pore architectures have been reported following this concept. ${ }^{119 a, 123}$ Some of these extra-large pore zeolites show multipore structures, offering unique "molecular traffic control" opportunities for chemical processes involving bulky and small molecules.

\section{1.- ITQ-15/IM-12}

The first publication on a zeolite with interconnected extra-large and large pores was reported by Corma et al. in the patent literature, and was named ITQ-15. ${ }^{124}$ The structure was published simultaneously by the groups of Mulhouse, as IM-12, ${ }^{119 b}$ and Valencia, as ITQ-15, ${ }^{119 a}$ and has received the IZA code UTL. The pore system of UTL consists of an extra-large 14-ring channel along the $c$ axis with openings of $10 \times 6.7 \AA$, intersected perpendicularly by a 12-ring channel, along the $b$ axis, with pore openings of $8.4 \times 5.8 \AA$ (see framework topology in Figure 18). ${ }^{119}$ In both cases, aluminum atoms can be introduced in the UTL frameworks, creating Brønsted acid sites for catalytic applications. Pyridine adsorption-desorption experiments indicate the presence of strong acid sites in ITQ-15, ${ }^{119 a}$ and the catalytic benefit of combining extra-large and large pores in the same structure can be observed in the dealkylation experiments of two molecules with different sizes, triisopropylbenzene (TIPB) and diisopropylbenzene (DIPB), when compared with a unidirectional extra-large pore zeolite, as UTD-1. ${ }^{119 a}$ The 
kinetic reaction rates for dealkylation of the bulkier TIPB molecule are similar for ITQ15 and UTD-1, since TIPB can only diffuse through the 14-ring channel present in both zeolites. In contrast, the dealkylation rate for DIPB is much higher for ITQ-15 because this smaller molecule can diffuse through 14- and 12-ring pores present in ITQ-15, while only through the unidimenional 14-ring pore in UTD-1 zeolite.

Extra-large pore UTL zeolite has also been tested for the bifunctional hydroisomerization of $n$-decane, ${ }^{125}$ which is a reaction whose product distribution is very sensitive to the diameter of the accessible pores, and for aromatic transformation/revalorization. ${ }^{126}$ Thus, Kirschhock et al. have reported that the UTL zeolite shows higher selectivity towards the bulky 3- and 4-ethyloctane isomers than other medium and large pore zeolites, during the hydroconversion of n-decane. ${ }^{125}$ On the other hand, Cejka et al. reported that UTL is highly selective to cymene with almost no formation of the undesired $n$-propyltoluene product for toluene alkylation with isopropyl alcohol. ${ }^{126}$ The formation of xylenes by toluene disproportionation and trimethylbenzene disproportionation/isomerization are also favored on UTL as compared to $\mathrm{BEA}$ and $\mathrm{MFI},{ }^{126}$ in agreement with the presence of extra-large pores in UTL.

\section{2.- ITQ-33}

The success in the synthesis of the bi-dimensional extra-large pore ITQ-15 motivated us to open new synthetic approaches to rationalize the preparation of more open multipore zeolites for their application as efficient catalysts. Previous experience using small and flexible OSDAs, as for instance hexamethonium, allowed us to synthesize zeolitic materials with different pore topologies thanks to the large number of conformations that these flexible OSDAs can take under particular synthesis conditions. ${ }^{112}$ Thus, very flexible and unselective OSDAs were combined with inorganic directing agents, such as Ge atoms and F anions, which are known to stabilize the formation of small SBUs, such as D4R or 3-rings. The objective was again the synthesis of multidimensional extra-large pore zeolites with interconnected pores. ${ }^{3}$ Thus, an experimental design was proposed combining the use of inorganic and organic structure directing agents, while using high-throughput synthesis methods. ${ }^{123 a, 127}$ Following this accelerated synthesis methodology, the synthesis was directed towards 
the crystallization of a new extra-large pore pure phase zeolite, named ITQ-33, from initial mixtures where ITQ-33 was present only as a minor phase. ${ }^{123 a, 127}$

The framework structure of ITQ-33 zeolite is very unique, presenting straight extralarge 18-ring channels with pore openings of $12.2 \AA$ (see Figure 18 and Figure 19c), interconnected by a bidirectional system of 10-ring channels with pore openings of 6.1 x $4.3 \AA$ (see Figure 19d). ${ }^{123 a}$ This very open three-dimensional pore topology presents 12.3 T-atoms per $1000 \AA^{3}$, which was the lowest FD ever reported for a crystalline zeolite at that moment. ITQ-33 could be prepared with framework aluminum, resulting in a material with Brønsted acidity. The presence of Brønsted acid sites combined with the particular framework topology of ITQ-33 opens attractive possibilities for catalysis. For instance, ITQ-33 shows very high activity for the industrially relevant alkylation of benzene with propylene to produce cumene (see Figure 20), giving an extremely low yield of undesirable $n$-propylbenzene (less than $0.01 \%$ yield). ${ }^{123 a, 127}$ As seen in Figure 20, ITQ-33 performs better and is more stable towards deactivation than commercial Beta zeolite. The lower catalyst deactivation of ITQ-33 observed for benzene alkylation can be explained by the presence of the extra-large 18-ring that pores favors the formation and diffusion of the alkylated products, while the presence of medium pores reduces the undesired propylene oligomerization. ${ }^{123 a, 127}$ Interestingly, ITQ-33 zeolite shows an interesting behavior for catalytic cracking of a vacuum gasoil (see Table 2). ${ }^{123 a, 127}$ ITQ-33 allows producing more diesel and less gasoline, while increasing propylene and butene yields than commercial catalysts based on USY zeolites (see USY and Beta catalytic activities in Table 2). Thus, with ITQ33 it was possible to simultaneously maximize the yields to propylene and diesel, being this catalytic behavior of significance because of the diesel production deficiency in European refineries, and the increasing demand of light olefins for chemical and petrochemical processes. ${ }^{128}$ The structure of ITQ-33 containing interconnected extralarge 18-ring pores and medium 10-ring pores is an adequate structure to maximize both selectivities simultaneously, since the very large pores allows the production of $C_{3}$ and $\mathrm{C}_{4}$ olefins by diffusion and cracking of gasoline molecules.

\section{3- Other extra-large germanosilicates with D3R in their structure (ITQ-40 and ITQ-} 44) 
The increase of the Ge content in the synthesis media (Si/Ge $\sim 0.7-1.2$ ) has recently allowed the synthesis of two multipore extra-large pore silicogermanates with the lowest framework densities ever reported for zeolitic materials, ITQ-40 (FD = 10.1 $\left.\mathrm{T} / 1000 \AA^{3}\right)^{123 e}$ and ITQ-44 (FD = $\left.10.9 \mathrm{~T} / 1000 \AA^{3}\right){ }^{123 \mathrm{c}}$ In both cases, the large Ge content directs to the formation not only of D4R, but also of double-3-rings (D3R) for the first time in crystalline zeolitic materials (see D3R in the structure of ITQ-44 in Figure 19e). ITQ-40 shows a three-dimensional framework with intersecting $16 \times 16 \times$ 15 -ring pores with crystallographic pore openings of $9.9 \AA$ for the circular 15 -ring pore running along the $c$ axis, and $9.4 \times 10.4 \AA$ pore openings for the two 16-ring pores running along the $a$ and $b$ axes. ${ }^{123 e}$ In contrast, ITQ-44 shows a three-dimensional framework with intersecting $18 \times 12 \times 12$-ring pores with pore openings of $12.5 \AA$ for the circular 18-ring pore along $c$ axis (see Figure $19 \mathrm{c}$ ), and $8.2 \times 6.0 \AA$ for the system of 12-ring pores along the plane $a-b$ (see Figure 19e). As it can be seen in Figure 19, the structure of ITQ-44 is closely related to ITQ-33. Both are formed from the same zeolitic layer (see Figure 19b), which is connected by simple 3-rings in the case of ITQ-33 and D3Rs in the case of ITQ-44 (see Figures 19d and 19e, respectively). The hydrothermal stability of these zeolites should be improved by reducing the Ge content in order to study their potential as catalysts in applications involving bulky molecules.

\section{6.- Conclusions and perspectives}

Along the present manuscript, the synthesis and the catalytic applications of the most interesting microporous molecular sieves containing interconnected pores with different dimensions have been described. The benefit of these multipore zeolites for particular catalytic applications has been shown, not only in terms of selective diffusion of reactants or products by "molecular traffic control" through the different sized channels, but also by the selective active site population in certain crystallographic positions of these different pores/cavities.

Despite the large number of multipore zeolites reported up to date and presented here, efforts are still required for improving synthesis procedures, including chemical compositions, hydrothermal stabilities, and synthesis costs.

One of the big challenges in the next years will be the synthesis of hydrothermally stable multipore zeolites containing extra-large pores. As described above, several 
extra-large multipore zeolites have been recently synthesized due to the introduction of Ge atoms as inorganic SDA. ${ }^{120}$ Unfortunately, these extra-large pore germanium-rich molecular sieves show low hydrothermal stabilities. Recently, some examples of postsynthetic isomorphic substitutions of $\mathrm{Ge}$ atoms in framework positions by $\mathrm{Al}$ or $\mathrm{Si}$ atoms have allowed improving the hydrothermal stability of these catalysts, ${ }^{111 a, 129}$ but up to now, these post-synthetic procedures have only been reported for large pore zeolites (i.e. ITQ-17 or ITQ-22). It is expected that the selective isomorphic substitution of Ge by other heteroatoms under the adequate conditions will allow the preparation of quasi Ge-free extra-large pore zeolites in the near future.

Since the synthesis of extra-large pore zeolites containing some specific small building units, such as D4R or D3R, is very difficult under Ge-free high-silica conditions, ${ }^{120}$ we predict that one of the most feasible ways to obtain hydrothermally stable high-silica extra-large pore zeolites would be as interrupted frameworks with ordered distribution of structural defects in the crystalline structure. The presence of framework defects in specific crystallographic positions could favor the formation of very open pores. In this sense, the design of bulky and specific OSDAs may be required to fill the large void volumes of extra-large multipore zeolites, and most importantly, to selectively locate framework defects by proper charge balance between the OSDAs and the siloxy species of the structural defects. The use of molecular modeling techniques could help designing and selecting the best OSDA candidates to template extra-large multipore zeolites.

Interestingly, linear and flexible OSDAs have also been able to direct the crystallization of different multipore zeolites. Particularly interesting is the case of the commercially available hexametonium cation, which directs the crystallization of multipore zeolites ranging from medium-small pores to extra-large pores (see Figure 21). The use of linear flexible OSDAs with bulkier substituents in the cationic heads may also favor the filling of the large void volumes required to template large or extra-large multipore zeolites under high-silica synthesis conditions.

The rational synthesis of ITQ-39 using bifunctional OSDAs with the appropriate rigidity and flexibility opens a very attractive methodology to direct the formation of new zeolites with interconnected large and medium pores. ${ }^{112-113}$ Since these types of multipore zeolites offer a unique opportunity to combine the catalytic properties of 
two important zeolites in industrial applications, such as Beta and ZSM-5, it could be predicted that the design of new OSDAs with adequate rigidity/flexibility will allow the synthesis of new zeolitic structures with interconnected large and medium pores.

Importantly, the costs associated to the synthesis of multipore zeolites must be competitive to consider their potential application in industrial processes. In this sense, the preparation of known multipore zeolites following an OSDA-free synthesis methodology, ${ }^{130}$ or using inexpensive OSDAs (i.e. amines) ${ }^{10 \mathrm{~b}}$ can be helpful.

In addition, the use of mixtures of OSDAs would not only allow improving the economy of the synthesis, if the original expensive OSDA is partially substituted by inexpensive organic molecules, but could also present a direct effect on the active site distribution within the pores/cavities. A preferential population of the active sites in different pores o crystallographic positions will have a clear impact on the catalytic activities and selectivities for particular chemical processes. ${ }^{18 \mathrm{~d}, 23}$

\section{Acknowledgements}

Financial support by the Spanish Government-MINECO through "Severo Ochoa" (SEV 2012-0267), Consolider Ingenio 2010-Multicat, MAT2012-37160, MAT2012-31657 and Intramural-2014801015 is acknowledged. 
Figure 1: Dimensions through a molecule can diffuse within the zeolitic crystals

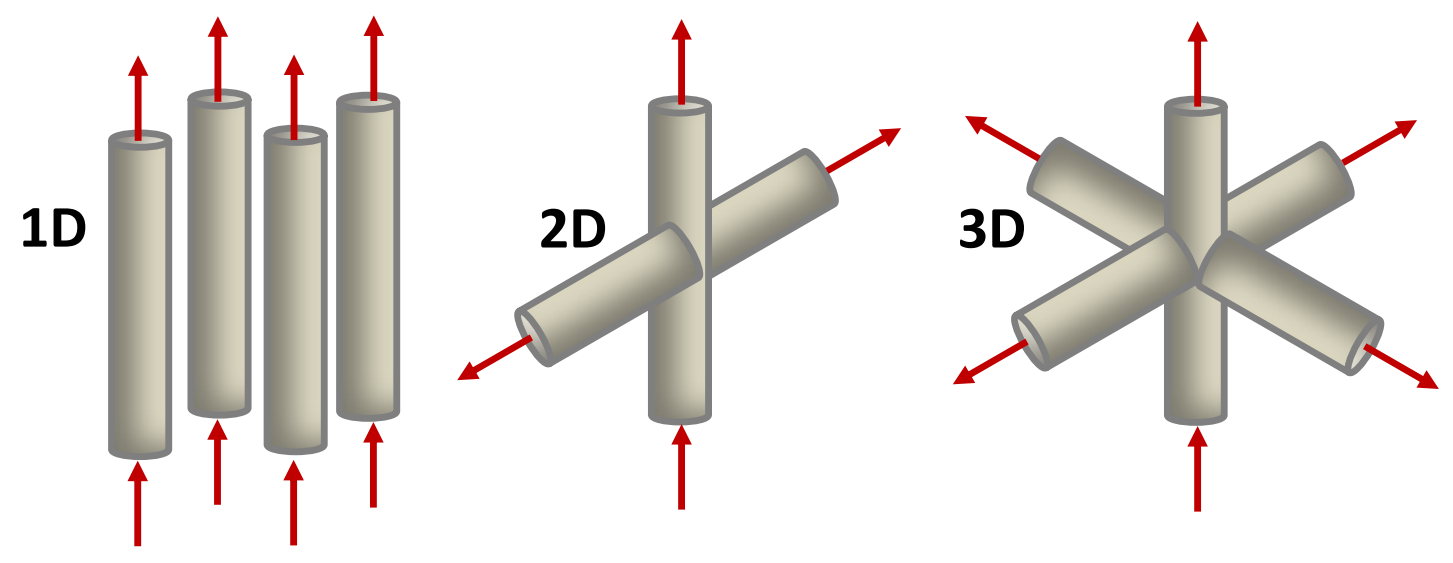


Figure 2: Scheme of the FER cavity (A) and FER assembly using cooperative OSDA agents (B). Reproduced from ${ }^{18 d}$.

A)
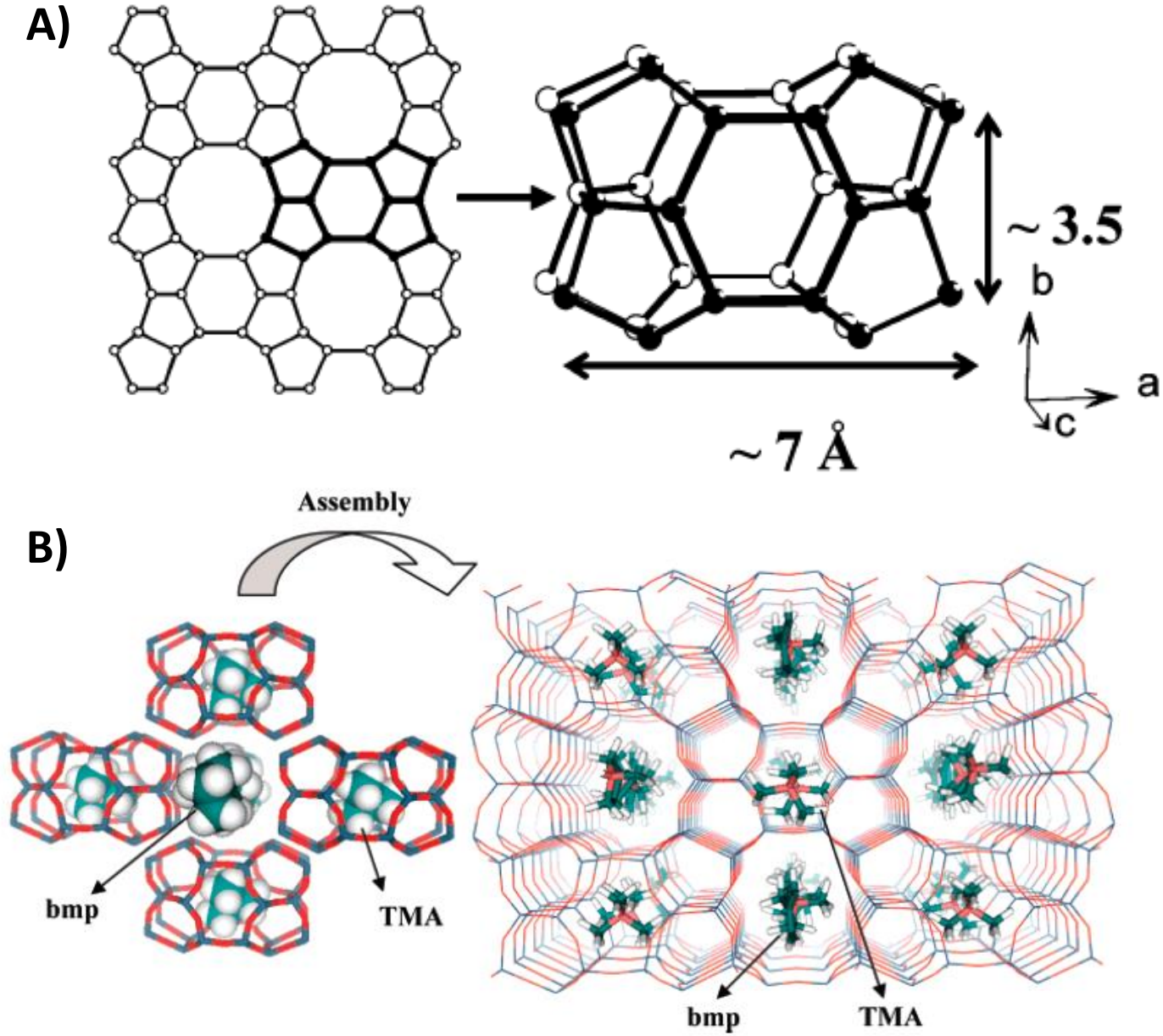
Figure 3: OSDA used for the synthesis of different zeolites presenting interconnected medium and small pores.

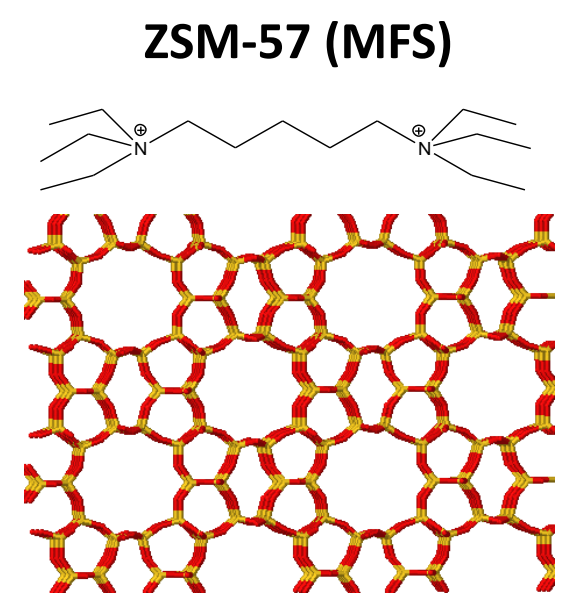

ITQ-13 (ITH)

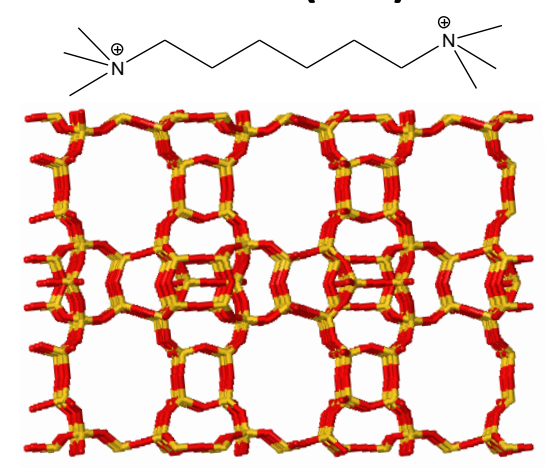

TNU-10 (STI)

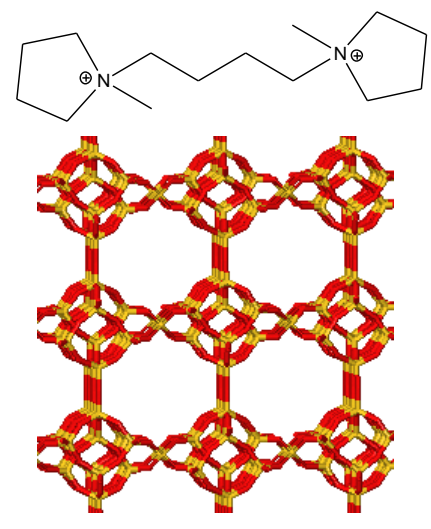

ITQ-34 (ITR)

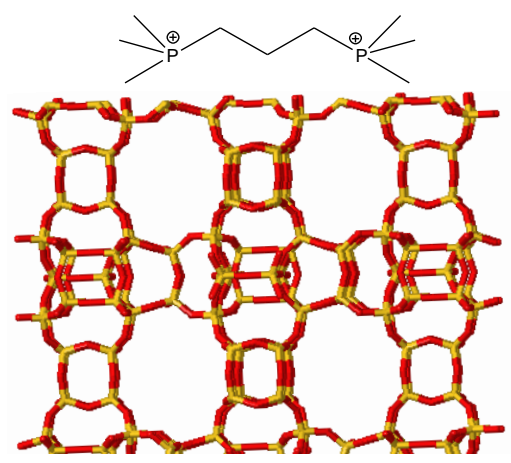


Figure 4: 1-Butene conversion (a) and isobutene selectivity (b) for the skeletal isomerization of 1-butene using FER (triangles) and TNU-10 (squares). Reproduced from ${ }^{34 b}$.
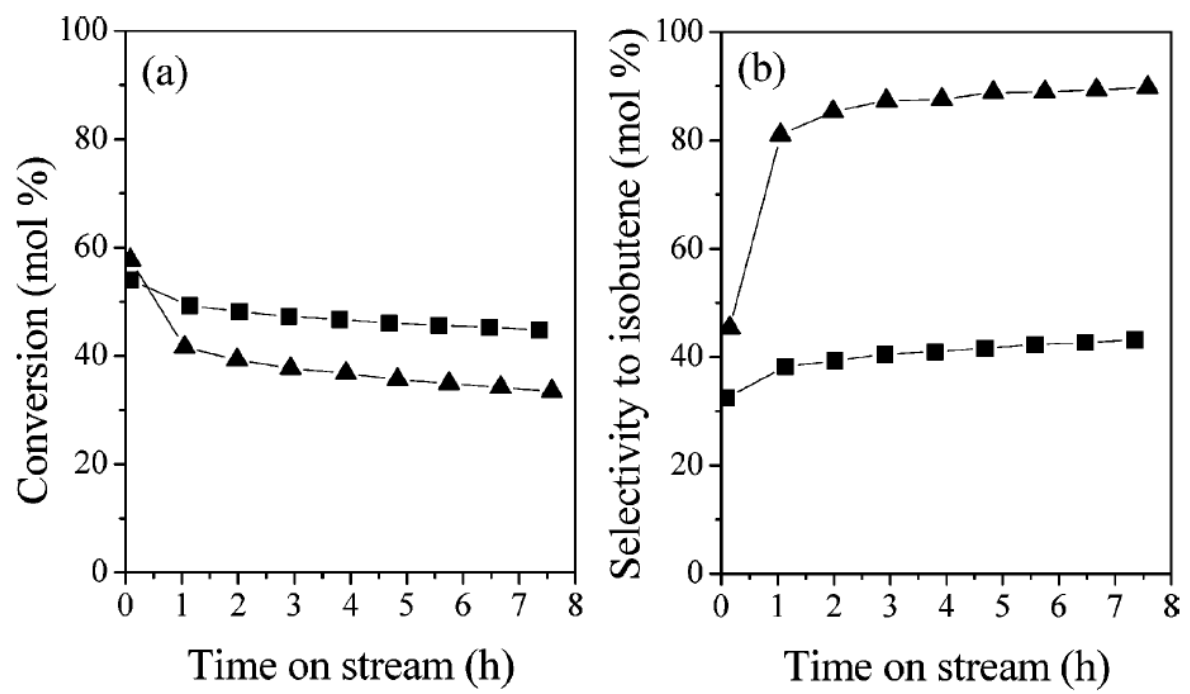
Figure 5: Structural similarities between SSZ-51(SFO) and SAPO-40 (AFR) zeotypes. Reproduced from ${ }^{56}$.
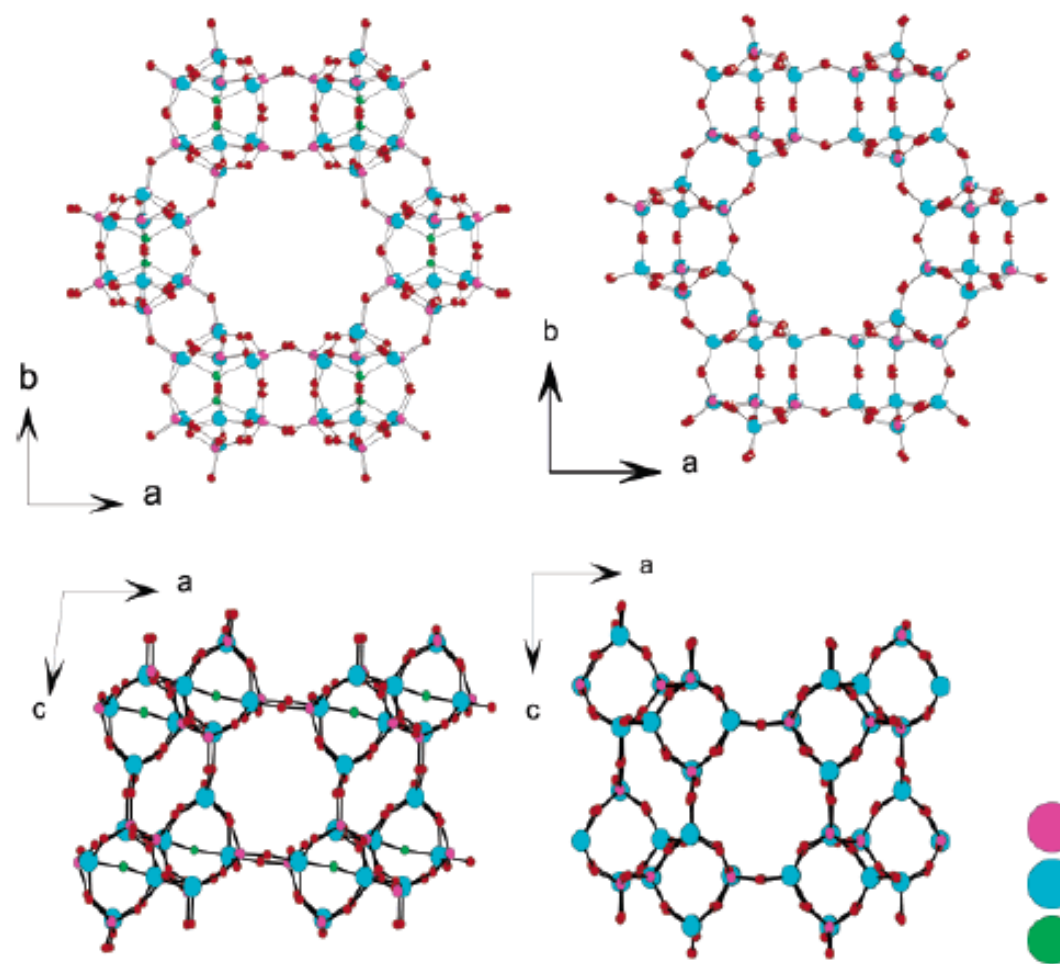

SSZ-51

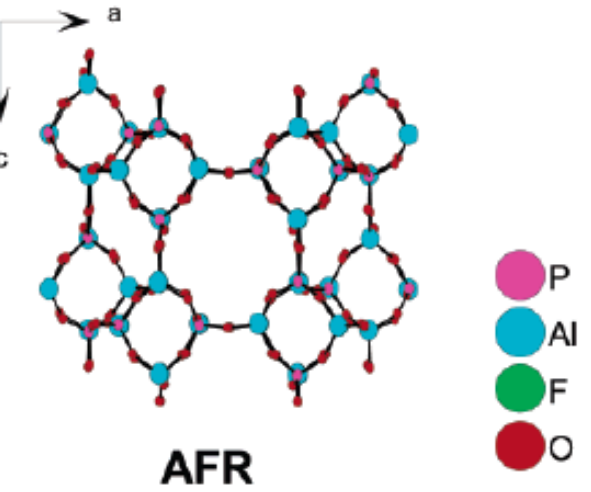


Figure 6: Structures of BPH, MOZ and OFF.
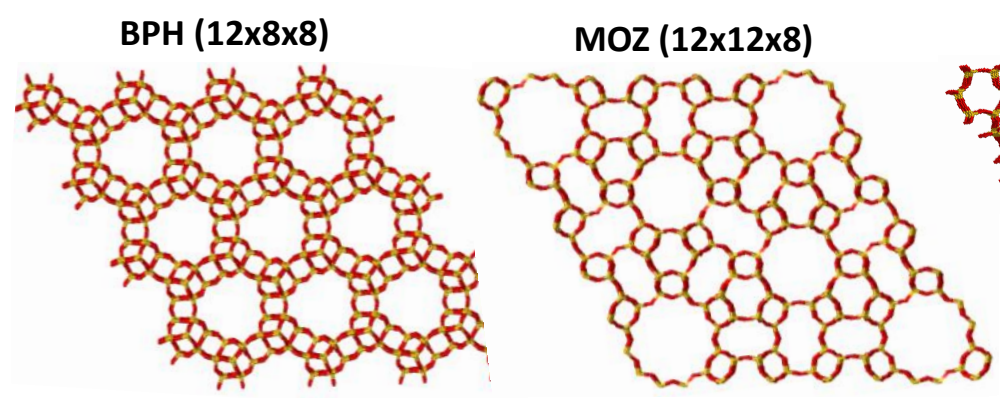
OFF (12x8)

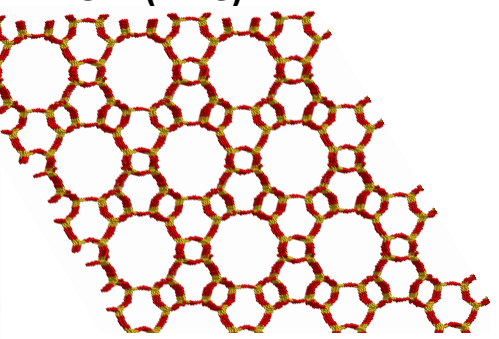


Figure 7: Views of MOR structure along the 12-ring pores $(A)$ and 8-ring pores (B), and different crystallographic positions for T atoms in MOR (C and D). Reproduced from ${ }^{17 c}$.

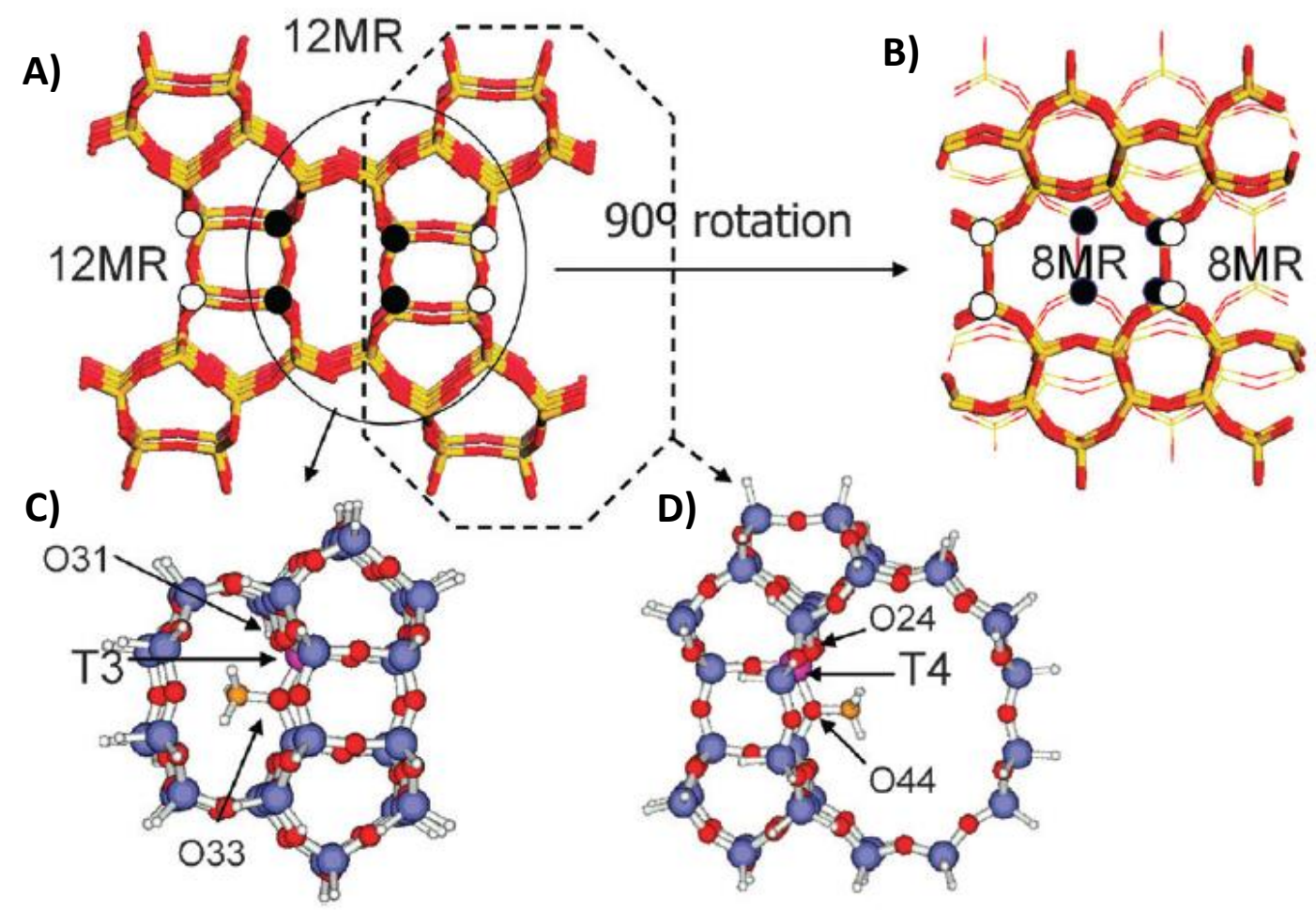


Figure 8: Framework structures of polymorph A (A), polymorph $B(B)$, and polymorph C (C) of the CON family viewed along the 10 -ring pores. Reproduced from ${ }^{86}$.
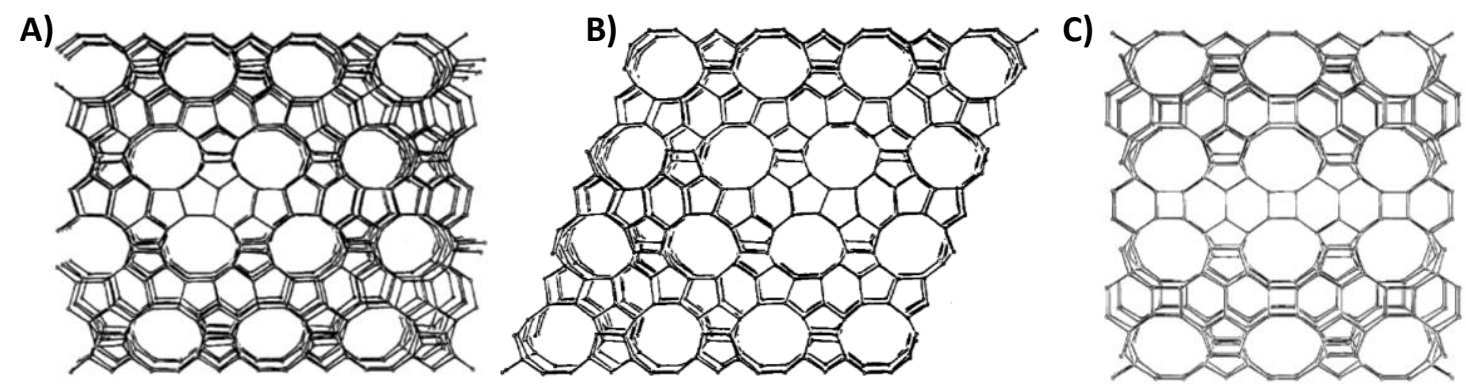
Figure 9: OSDAs used for the synthesis of SSZ-26 (a), and SSZ-33 (b), and OSDA distribution within the SSZ-26 (c). Reproduced from ${ }^{86}$.

A)

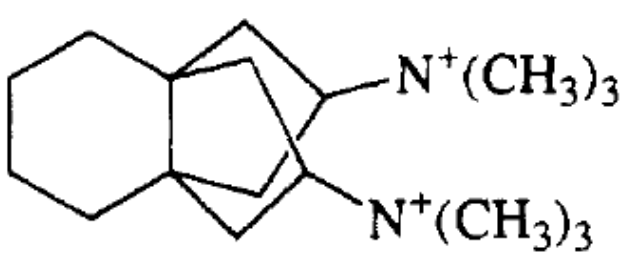

B)

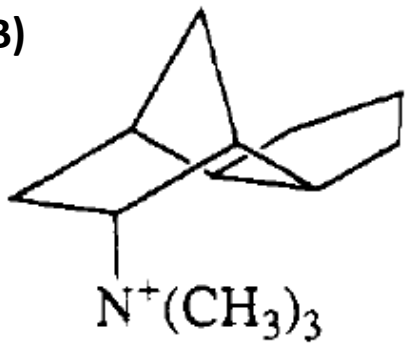

C)

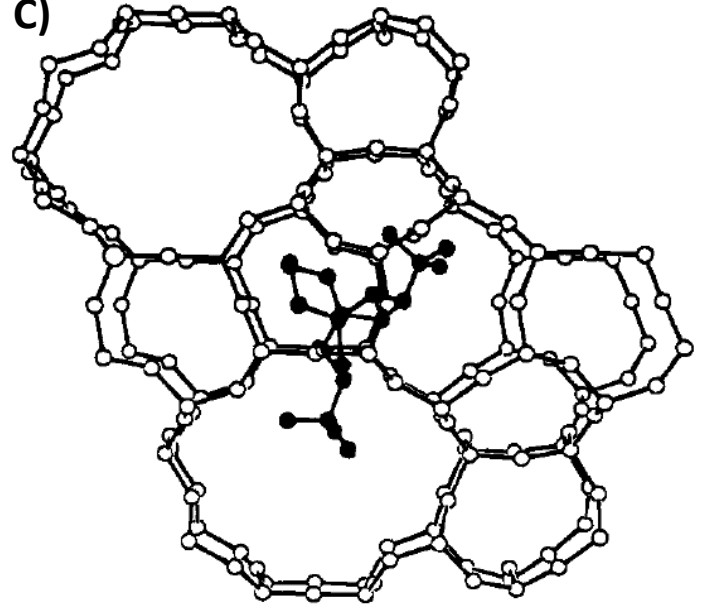


Figure 10: OSDAs used for the synthesis of MCM-68 (A), ITQ-24 (B), and ITQ-47 (C).

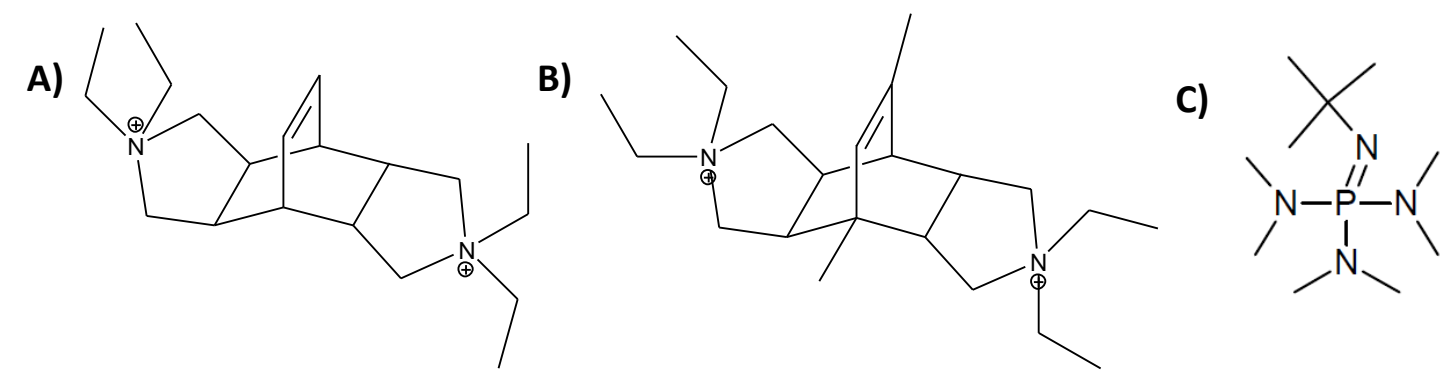


Figure 11: $p$-xylene/o-xylene (A) and isomerization/disproportionation (B) ratios for different zeolites attending to window sizes. Reproduced from ${ }^{91 a}$.
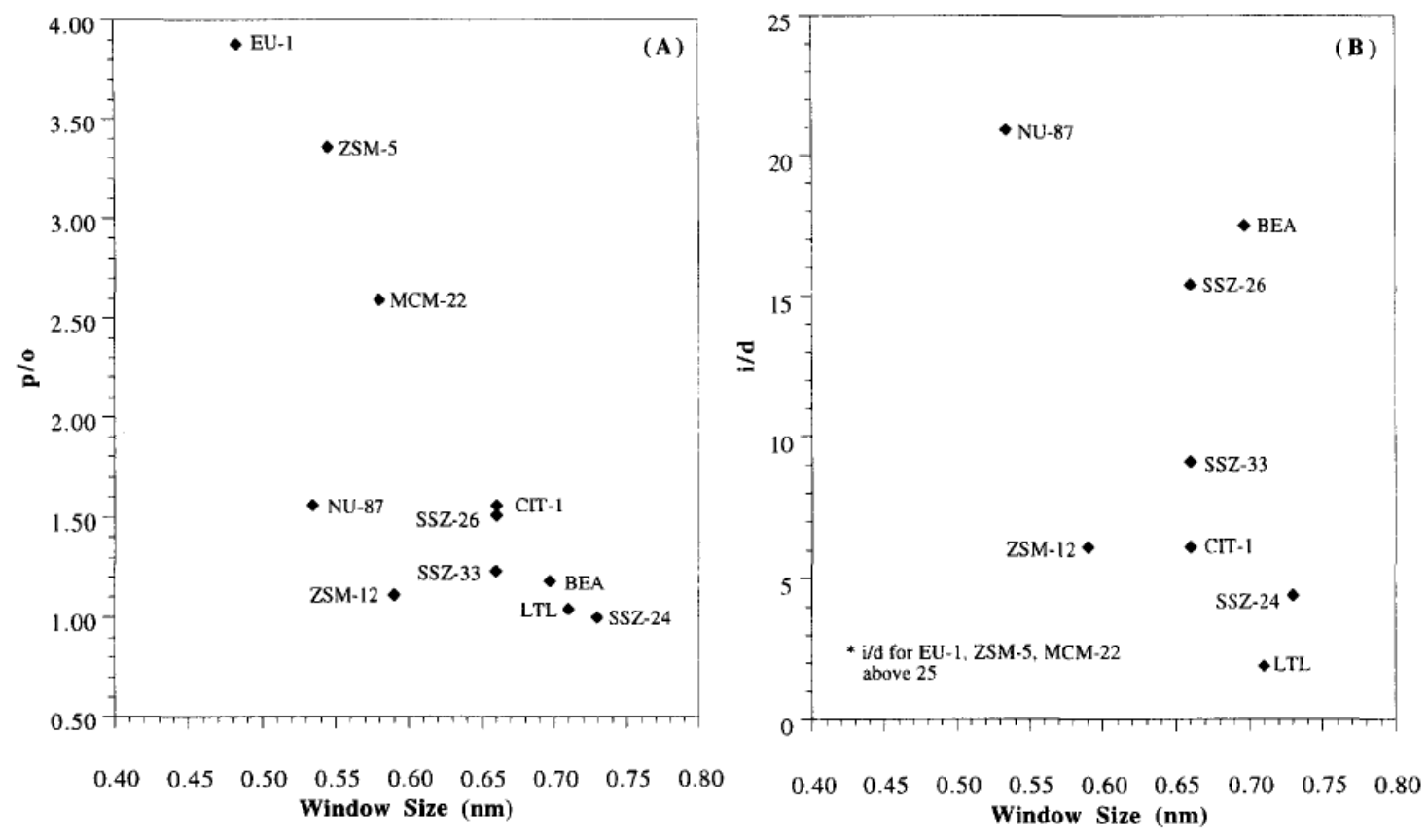
Figure 12: Zeolitic frameworks of SSZ-56 (A), SSZ-82 (B), and SSZ-57 (C), and the corresponding OSDAs used for their syntheses.

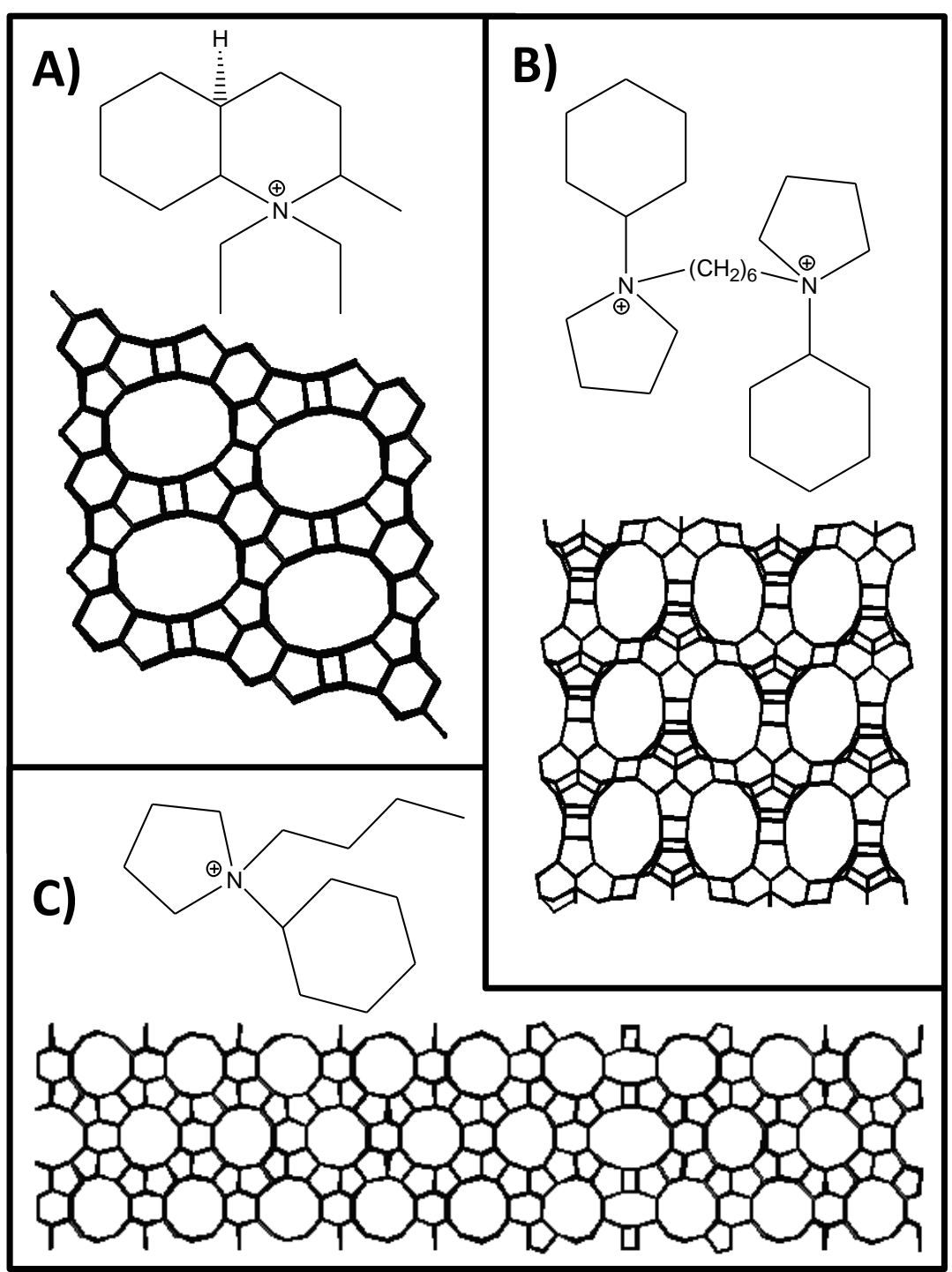


Figure 13: Structure model of ITQ-22 showing the 8- and 12-ring pores intersected by the sinusoidal 10-ring pore (black ribbon). Reproduced from ${ }^{110}$.

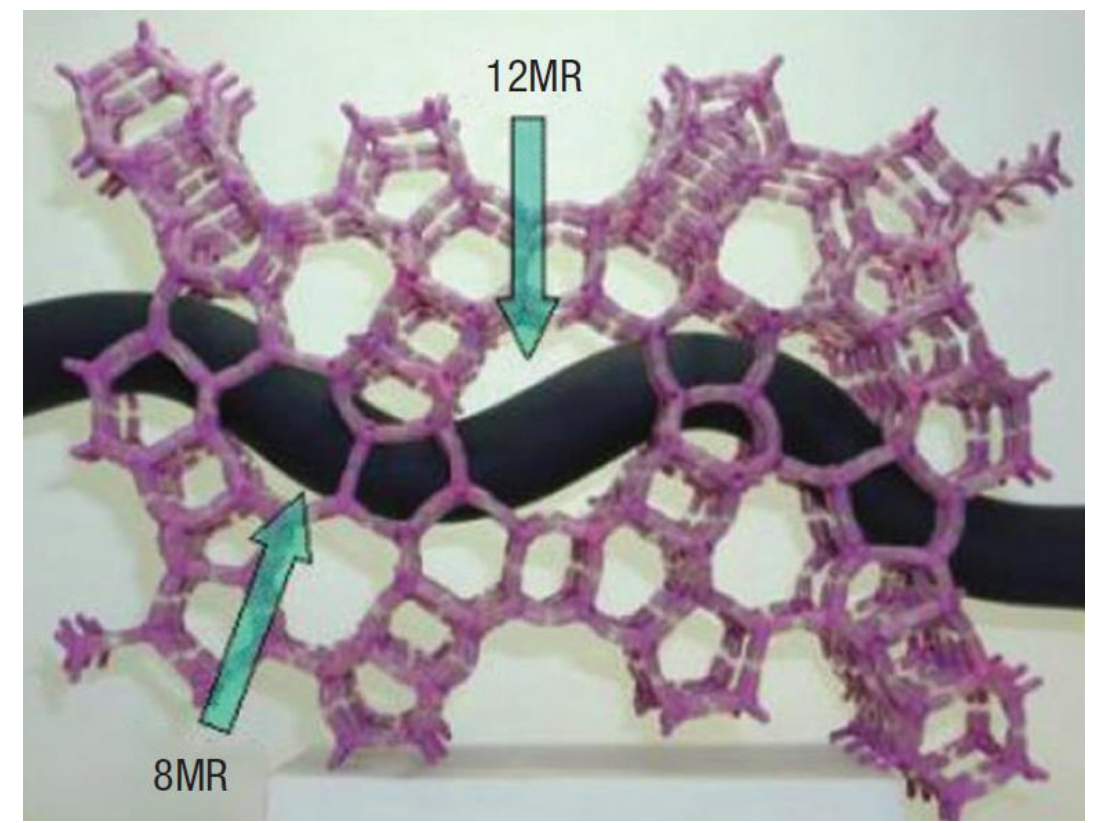


Figure 14: Benzene conversion (A) and ethylbenzene selectivity (B) for benzene alkylation with ethanol for ZSM-5 (squares), ITQ-22 (circles), SSZ-33 (triangles), and Beta (crosses). Reproduced from ${ }^{15 f}$.
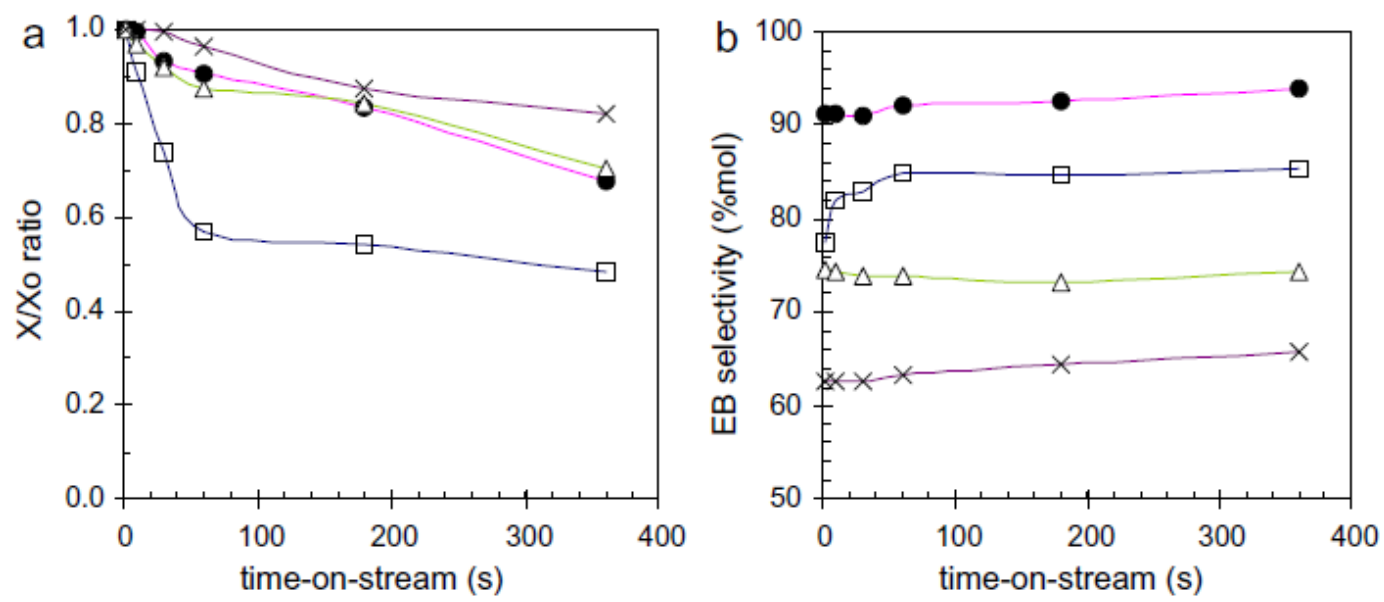
Figure 15: (A) Multifunctional OSDA for the direct synthesis of zeolites with interconnected large and medium pores. B) multipore structure of ITQ-39 zeolite. Reproduced from ${ }^{84,112}$.

A)<smiles>CCN1CCCC1C1CCCCC1</smiles>

ZSM-12 (12-ring)

$+$

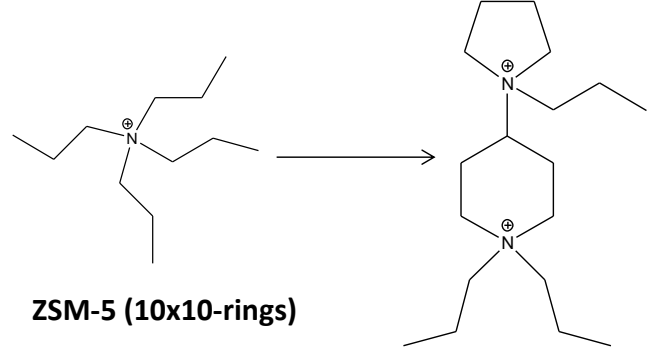

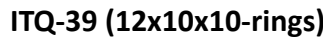

B)

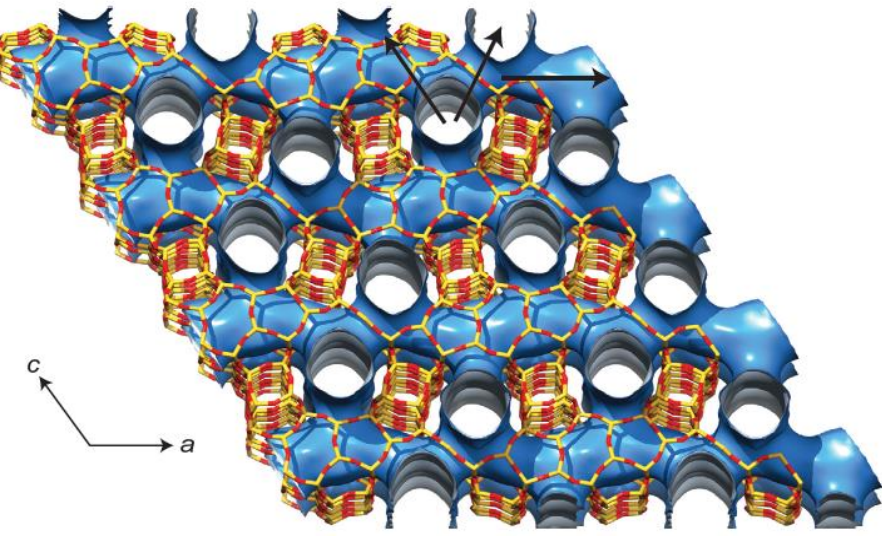


Figure 16: Comparison of the catalytic results of ITQ-39, Beta and MWW for alkylation of naphtha with olefins. Reproduced from ${ }^{84,112}$.
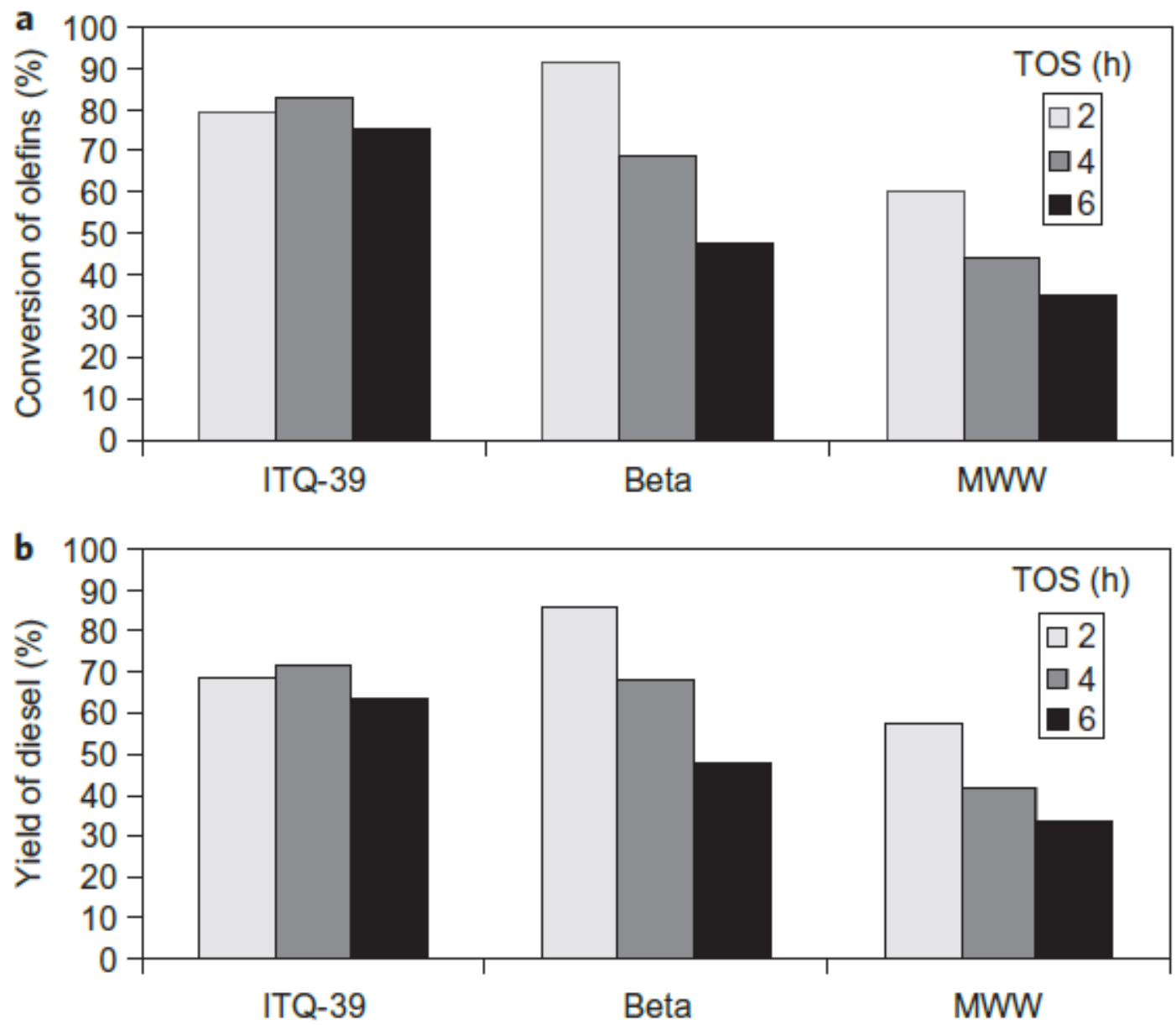
Figure 17: Zeolites with interconnected large and medium pores synthesized from layered precursors: APZ-4 from PREFER (A), and COK-14 from UTL (B). Reproduced from ref ${ }^{117}$ and ${ }^{118}$ respectively.

A)

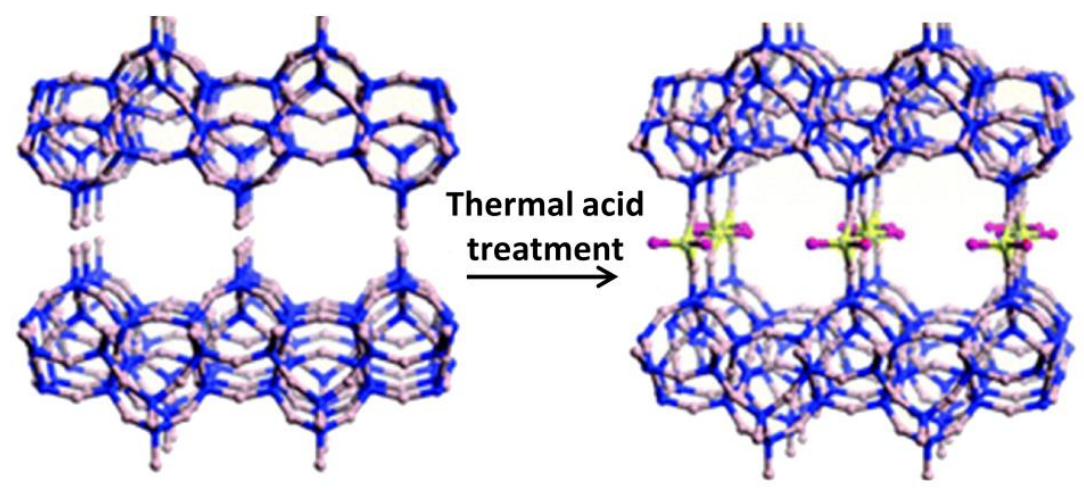

B)

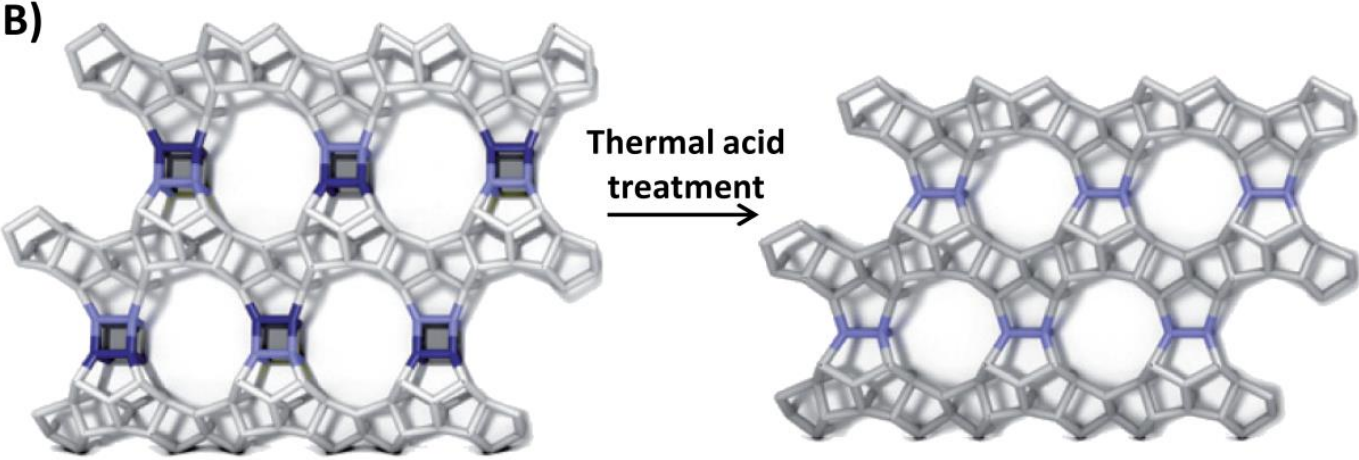


Figure 18: Structures of multipore extra-large pore zeolites.

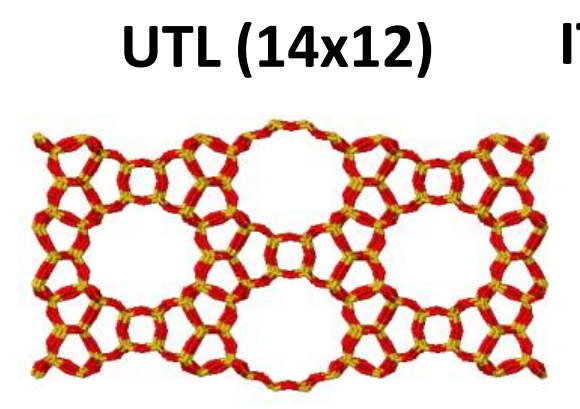

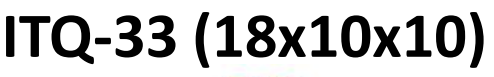

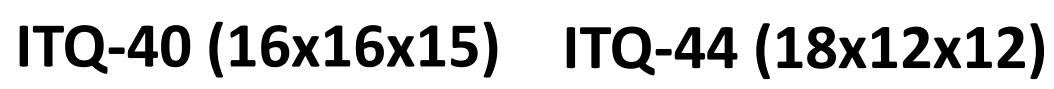
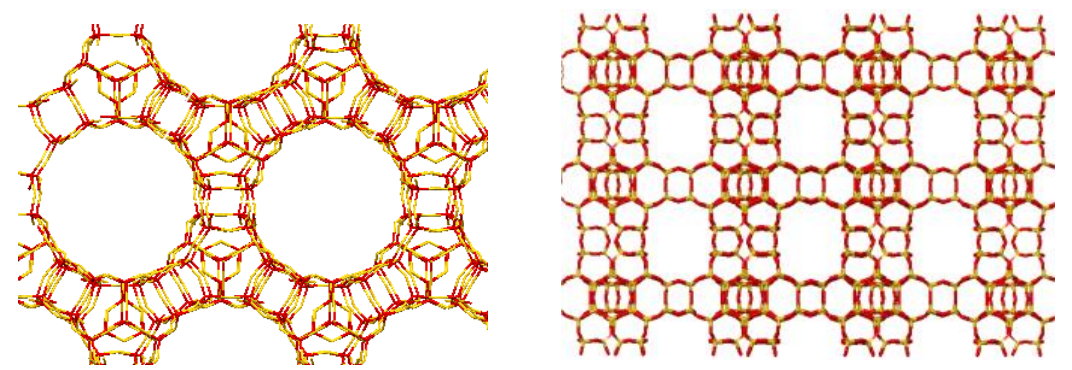
Figure 19: (a) Common building unit of ITQ-33 and ITQ-44, (b) common zeolitic layer for ITQ-33 and ITQ-44, (c) view of the 18-ring pores of ITQ-33 and ITQ-44, (d) view of the 10-ring pores of ITQ-33, (e) view of the 12-ring pores of ITQ-44. Reproduced from $123 c$.

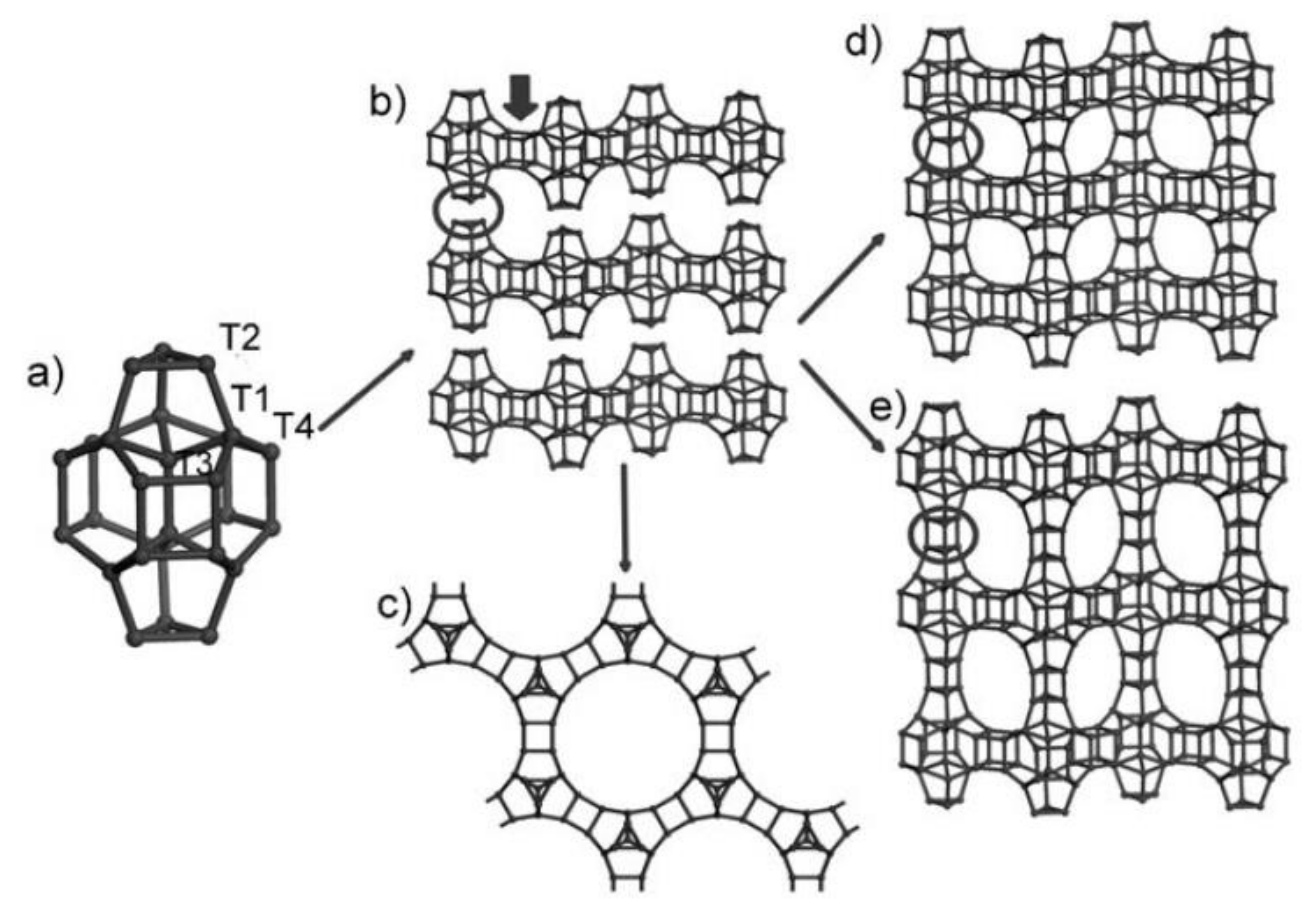


Figure 20: Propylene conversion using ITQ-33 (filled circles) and Beta (open circles). Reproduced from ${ }^{123 a}$.

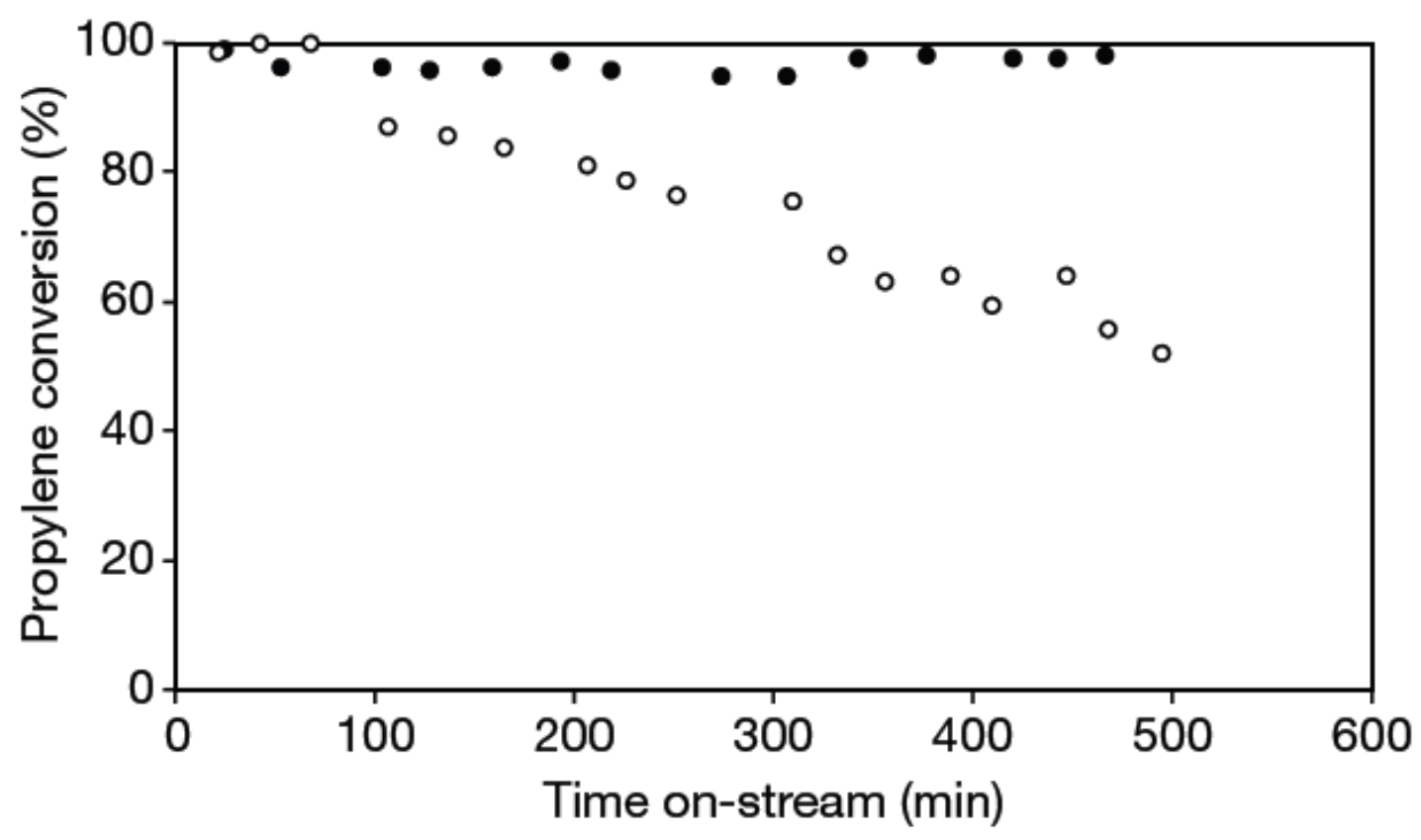


Figure 21: Different multipore zeolites synthetized using hexamethonium as OSDA.

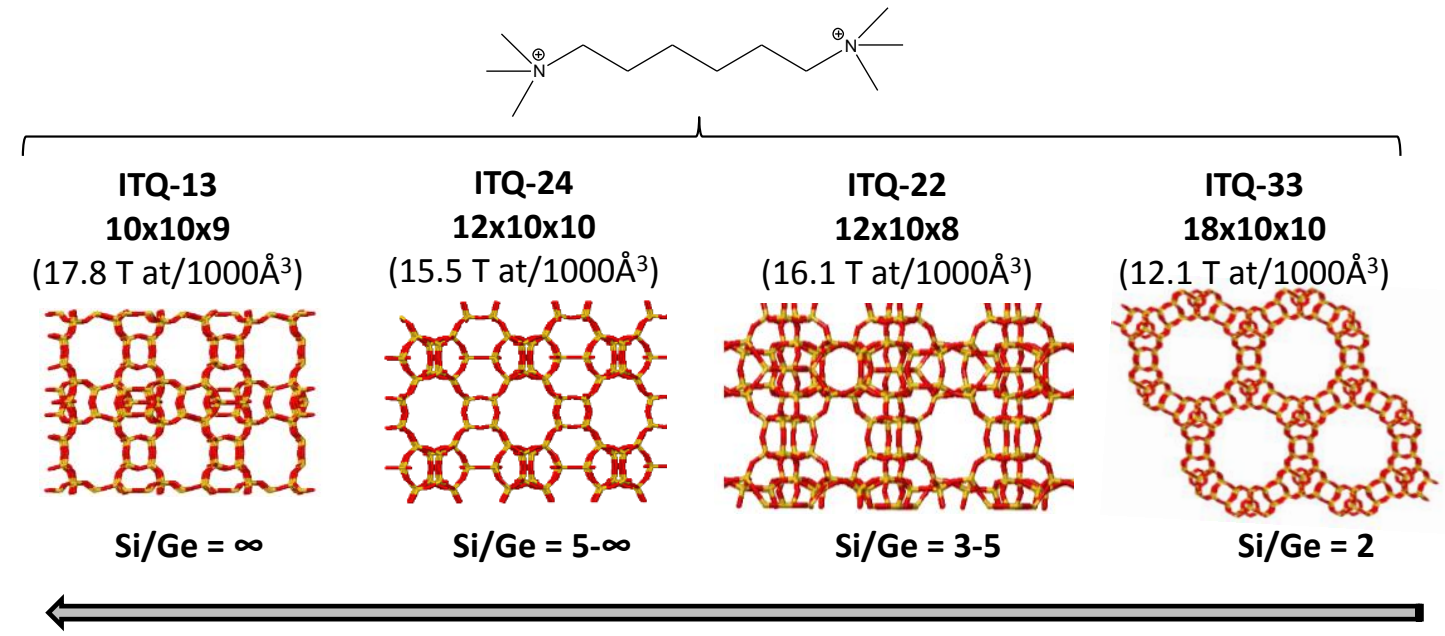

个 Si/Ge 
Table 1: Zeolites presenting multipore systems

\begin{tabular}{|c|c|c|}
\hline IZA code & Zeolite Names & Pore architecture \\
\hline AFR & SAPO- $40^{52}$ & $12 \times 8$ \\
\hline AFS & MAPSO- $46^{131}$ & $12 \times 8$ \\
\hline AFY & CoAIPO- $50^{58}$ & $12 \times 8 \times 8$ \\
\hline $\mathrm{BPH}$ & UZM-4, ${ }^{61}$ STA- $5^{59}$ & $12 \times 8 \times 8$ \\
\hline BOG & ITQ- $47^{95}$ & $12 \times 10$ \\
\hline CGF & Co-Ga-phosphate- $5^{132}$ & $10 \times 8$ \\
\hline CGS & $\begin{array}{c}\text { Co-Ga-phosphate-5, } \\
\text { TNU-1 }{ }^{134}\end{array}$ & $10 \times 8 \times 8$ \\
\hline CON & CIT-1, ${ }^{87}$ SSZ-26, ${ }^{85}$ SSZ-33 ${ }^{85}$ & $12 \times 12 \times 10$ \\
\hline EON & ECR-1 ${ }^{135}$ & $12 \times 8 \times 8$ \\
\hline ETR & ECR-34 ${ }^{136}$ & $18 \times 8 \times 8$ \\
\hline FER & Ferrierite $^{137}$ & $10 \times 8$ \\
\hline IRR & ITQ-44 ${ }^{123 c}$ & $18 \times 12 \times 12$ \\
\hline ITG & ITQ-38 & $12 \times 10 \times 10$ \\
\hline ITH & ITQ $-13^{37}$ & $10 \times 10 \times 9$ \\
\hline ITR & ITQ-34 & $10 \times 10 \times 9$ \\
\hline ITT & ITQ-33 ${ }^{123 a}$ & $18 \times 10 \times 10$ \\
\hline IWR & ITQ-24 ${ }^{89}$ & $12 \times 10 \times 10$ \\
\hline IWW & ITQ-22 $2^{110}$ & $12 \times 10 \times 8$ \\
\hline MFS & $\mathrm{ZSM}-57^{138}$ & $10 \times 8$ \\
\hline MOR & Mordenite ${ }^{73}$ & $12 \times 8$ \\
\hline $\mathrm{MOZ}$ & $\mathrm{ZSM}-10^{62}$ & $12 \times 12 \times 8$ \\
\hline MSE & MCM-68 ${ }^{96}$ & $12 \times 10 \times 10$ \\
\hline OFF & Offretite $^{139}$ & $12 \times 8$ \\
\hline OKO & COK-14, ${ }^{118}$ IPC- $2^{51}$ & $12 \times 10$ \\
\hline PCR & IPC- $4^{51}$ & $10 \times 8$ \\
\hline RRO & RUB-41 ${ }^{46}$ & $10 \times 8$ \\
\hline SBE & UCSB-8 $8^{60}$ & $12 \times 8$ \\
\hline
\end{tabular}




\begin{tabular}{|c|c|c|}
\hline SBS & UCSB-6 ${ }^{60}$ & $12 \times 12 \times 8$ \\
\hline SBT & UCSB-10 $0^{60}$ & $12 \times 12 \times 8$ \\
\hline SEW & SSZ-82 $2^{103}$ & $12 \times 10$ \\
\hline SFO & SSZ-51 $1^{56}$ & $12 \times 8$ \\
\hline SFS & SSZ-56 $6^{102}$ & $12 \times 10$ \\
\hline SFV & SSZ-57 & $12 \times 10 \times 10$ \\
\hline STI & TNU-10 $34 \mathrm{~b}$ & $10 \times 8$ \\
\hline SZR & SUZ-4 ${ }^{140}$ & $10 \times 8 \times 8$ \\
\hline UOS & $\mathrm{IM}-16^{141}$ & $10 \times 8 \times 8$ \\
\hline UTL & IM-12, ${ }^{119 b}$ ITQ-15 $5^{119 a}$ & $14 \times 12$ \\
\hline UWY & $\mathrm{IM}-20^{142}$ & $12 \times 10$ \\
\hline --- & EMM- $8^{57}$ & $12 \times 8$ \\
\hline --- & ITQ-3984 & $12 \times 10 \times 10$ \\
\hline
\end{tabular}


Table 2: Catalytic cracking of arabian light vacuum gasoil at 500 ㄷ

\begin{tabular}{|c|c|c|c|c|c|c|}
\hline Catalyst & X (\%) & $\begin{array}{c}\text { Diesel } \\
\text { yield } \\
\mathbf{( \% )}\end{array}$ & $\begin{array}{c}\text { Gasoline } \\
\text { yield } \\
\mathbf{( \% )}\end{array}$ & $\begin{array}{c}\text { Propylene } \\
\text { yield } \\
\mathbf{( \% )}\end{array}$ & $\begin{array}{c}\text { Propylene } \\
\text { /propane }\end{array}$ & $\begin{array}{c}\text { Isobutene/ } \\
\text { isobutane }\end{array}$ \\
\hline USY & 92.5 & 15.7 & 40.4 & 4.7 & 1.0 & 0.1 \\
\hline ITQ-33 & 89.2 & 22.6 & 34.5 & 4.2 & 1.9 & 0.4 \\
\hline Beta & 84.0 & 14.1 & 32.3 & 7.5 & 1.9 & 0.5 \\
\hline USY + 20\% ZSM-5 & 87.0 & 17.0 & 33.2 & 7.2 & 1.5 & 0.3 \\
\hline ITQ-33 + 20\% ZSM-5 & 86.1 & 23.3 & 25.1 & 9.0 & 3.7 & 1.1 \\
\hline
\end{tabular}




\section{REFERENCES}

(1) Cundy, C.; Cox, P. A. Micropor. Mesopor. Mater. 2005, 82, 1.

(2) (a) Davis, M. E. Nature 2002, 417, 813. (b) Corma, A. J. Catal. 2003, 216, 298. (c) Martínez, C.; Corma, A. Coord. Chem. Rev. 2011, 255, 1558. (d) Cejka, J.; Centi, G.; Perez-Pariente, J.; Roth, W. J. Catal. Today 2012, 179, 2.

(3) Corma, A.; Davis, M. E. ChemPhysChem 2004, 5, 304.

(4) Davis, M. E.; Lobo, R. F. Chem. Mater. 1992, 4, 756.

(5) Moliner, M.; Martínez, C.; Corma, A. Chem. Mater. 2014, 26, 246.

(6) (a) Corma, A.; Nemeth, L. T.; Renz, M.; Valencia, S. Nature 2001, 412, 423. (b) Moliner, M.; Corma, A. Micropor. Mesopor. Mater. 2014, 189, 31. (c) Notari, B. Catal. Today 1993, 18, 163.

(7) (a) Renz, M.; Blasco, T.; Corma, A.; Fornes, V.; Jensen, R.; Nemeth, L. T. Chem.-Eur. J. 2002, 8, 4708. (b) Corma, A.; Domine, M. E.; Nemeth, L. T.; Valencia, S. J. Am. Chem. Soc. 2002, 124, 3194.

(8) (a) Corma, A.; Iborra, S.; Velty, A. Chem. Rev. 2007, 107, 2411. (b) Roman-Leshkov, Y.; Davis, M. E. ACS Catal. 2011, 1, 1566. (c) Moliner, M. Dalton Trans. 2014, 43, 4197.

(9) (a) Wilson, S. T.; Lok, B. M.; Messina, C. A.; Cannan, T. R.; Flanigen, E. M. J. Am. Chem. Soc. 1982, 104, 1146. (b) Pastore, H. O.; Coluccia, S.; Marchese, L. Ann. Rev. Mater. Res. 2005, 35, 351. (c) Hartmann, M.; Kevan, L. Res. Chem. Inter. 2002, 28, 625.

(10) (a) Corma, A. Chem. Rev. 1997, 97, 2373. (b) Zones, S. I. Micropor. Mesopor. Mater. 2011, 144, 1.

(11) Csicsery, S. M. Zeolites 1984, 4, 202.

(12) Derouane, E. G. Stud. Surf. Sci. Catal. 1984, 19, 1.

(13) Derouane, E. G.; Gabelica, Z. J. Catal. 1980, 65, 486.

(14) (a) Lowe, B. W.; Whan, D. A.; Spencer, M. S. J. Catal. 1981, 70, 237. (b) Derouane, E. G.; Gabelica, Z.; Jacobs, P. A. J. Catal. 1981, 70, 238. (c) Pope, C. G. J. Catal. 1981, 72, 174. (d) Derouane, E. G. J. Catal. 1981, 72, 177. (e) Derouane, E. G. Appl. Catal. A 1994, 115, N2.

(15) (a) Clark, L. A.; Ye, G. T.; Snurr, R. Q. Phys. Rev. Lett. 2000, 84, 2893. (b) Neugebauer, N.; Braüer, P.; Kärger, J. J. Catal. 2000, 194, 1. (c) Brzank, A.; Schütz, G. Appl. Catal. A 2005, 288, 194. (d) Harish, R.; Karevski, D.; Schütz, G. M. J. Catal. 2008, 253, 191. (e) Chatterjee, S.; Harish, R.; Schütz, G. M. Chem. Ingen. Tech. 2013, 85, 1671. (f) Corma, A.; Llopis, F. J.; Martinez, C.; Sastre, G.; Valencia, S. J. Catal. 2009, 268, 9.

(16) (a) Gounder, R.; Iglesia, E. J. Am. Chem. Soc. 2009, 131, 1958. (b) Anquetil, R.; Saussey, J.; Lavalley, J. C. Phys. Chem. Chem. Phys. 1999, 1, 555. (c) Boronat, M.; MartinezSanchez, C.; Law, D.; Corma, A. J. Am. Chem. Soc. 2008, 130, 16316.

(17) (a) Bhan, A.; Allian, A. D.; Sunley, G. J.; Law, D. J.; Iglesia, E. J. Am. Chem. Soc. 2007, 129, 4919. (b) Bhan, A.; Iglesia, E. Acc. Chem. Res. 2008, 41, 559. (c) Boronat, M.; Martínez, C.; Corma, A. Phys. Chem. Chem. Phys. 2011, 13, 2603.

(18) (a) Guo, G.; Sun, Y.; Long, Y. Chem. Commun. 2000, 1893. (b) Schreyeck, L.; Caullet, P.; Mougenel, J. C.; Guth, J. L.; Marler, B. Microporous Mater. 1996, 6, 259. (c) Kim, T. J.; Ahn, W. S.; Hong, S. B. Microporous Mater. 1996, 7, 35. (d) Pinar, A. B.; GomezHortigüela, L.; Pérez-Pariente, J. Chem. Mater. 2007, 19, 5617.

(19) (a) Inaoka, W.; Kasahara, S.; Fukushima, T.; Igawa, K. Stud. Surf. Sci. Catal. 1990, 60, 37.

(b) Rakoczy, R. A.; Breuninger, M.; Hunger, M.; Traa, Y.; Weitkamp, J. Chem. Eng. Technol. 2002, 25, 273.

(20) Barrer, R. M.; Marshall, D. J. J. Chem. Soc. 1964, 485.

(21) (a) Song, J.; Dai, L.; Ji, Y.; Xiao, F. S. Chem. Mater. 2006, 18, 2775. (b) Xie, B.; Song, J.; Ren, L.; Ji, Y.; Li, J.; Xiao, F. S. Chem. Mater. 2008, 20, 4533. (c) Kamimura, Y.; Tanahashi, S.; Itabashi, K.; Shimojima, A.; Okubo, T. J. Phys. Chem. C 2011, 115, 744. 
(22) Gomez-Hortigüela, L.; Pinar, A. B.; Corà, F.; Pérez-Pariente, J. Chem. Commun. 2010, 46, 2073.

(23) Pinar, A. B.; Márquez-Álvarez, C.; Grande-Casas, M.; Pérez-Pariente, J. J. Catal. 2009, 263, 258.

(24) Roman-Leshkov, Y.; Moliner, M.; Davis, M. E. J. Phys. Chem. C 2011, 115, 1096.

(25) Mooiweer, H. H.; de Jong, K. P.; Kraushaar-Czarnetzki, B.; Stork, W. H. J.; Krutzen, B. C. H. Stud. Surf. Sci. Catal. 1994, 84C, 2327.

(26) (a) Szabo, J.; Perrotey, J.; Szabo, G.; Duchet, J. C.; Cornet, D. J. Phys. Chem. A 1991, 67, 79. (b) Houzvicka, J.; Ponec, V. Catal. Rev. Sci. Eng. 1997, 39, 319.

(27) http://www.epa.gov/mtbe/gas.htm.

(28) (a) Guisnet, M.; Andy, P.; Gnep, N. S.; Travers, C.; Benazzi, E. J. Chem. Soc., Chem. Commun. 1995, 1685. (b) de Menorval, B.; Ayrault, P.; Gnep, N. S.; Guisnet, M. J. Catal. 2005, 230, 38.

(29) (a) Pellet, R. J.; Casey, D. G.; Huang, H. M.; Kessler, R. V.; Kuhlman, E. J.; Oyoung, C. L.; Sawicki, R. A.; Ugolini, J. R. J. Catal. 1995, 157, 423. (b) Andy, P.; Gnep, N. S.; Guisnet, M.; Benazzi, E.; Travers, C. J. Catal. 1998, 173, 322.

(30) (a) Abrevaya, H.; Abdo, S. F.; Patton, R. L. U.S.Patent 6867341, 2005. (b) Abrevaya, H. Stud. Surf. Sci. Catal. 2007, 170, 1244. (c) Abrevaya, H.; P., T. G. U.S. Patent 2010/0105974, 2010. (d) Bortnovsky, O.; Sazama, P.; Wichterlova, B. Appl. Catal. A 2005, 287, 203. (e) Anderson, B. G.; Schumacher, R. R.; Van Duren, R.; Singh, A. P.; Van Saten, R. A. J. Mol. Catal. A 2002, 181, 291. (f) Bastiani, R.; Lam, Y. L.; Henriques, C. A.; da Silva, V. T. Fuel 2013, 107, 680.

(31) (a) Ernst, S.; Weitkamp, J. Stud. Surf. Sci. Catal. 1991, 65, 645. (b) Schlenker, J. L.; Higgins, J. B.; W., V. E. Zeolites 1990, 10, 293.

(32) Valyocsik, E. W.; Page, N. M. Eur.Pat. Appl. 174121, 1986.

(33) Martens, J. A.; Ravishankar, R.; Mishin, I. E.; Jacobs, P. A. Angew. Chem., Int. Ed. 2000, 39, 4376.

(34) (a) Galli, E.; Alberti, A. Bull. Soc. Fr. Mineral Cristallogr. 1975, 98, 331. (b) Hong, S. B.; Lear, E. G.; Wright, P. A.; Zhou, W.; Cox, P. A.; Shin, C. H.; Park, J. H.; Nam, I. S. J. Am. Chem. Soc. 2004, 126, 5817.

(35) (a) Liou, J. G. Contrib. Mineral. Petrol. 1971, 31, 171. (b) Ghobarkar, H.; Schaef, O.; Guth, U. J. Solid State Chem. 1999, 142, 451.

(36) (a) Zones, S. I.; Burton, A. W. U.S.Patent 7713512, 2010. (b) Davis, T. M.; Chen, C. Y.; Zilkova, N.; Vitvarova-Prochazkova, D.; Cejka, J.; Zones, S. I. J. Catal. 2013, $298,84$.

(37) Corma, A.; Puche, M.; Rey, F.; Sankar, G.; Teat, S. J. Angew. Chem., Int. Ed. 2003, 42, 1156.

(38) Boix, T.; Puche, M.; Camblor, M. A.; Corma, A. US 6,471,941, 2002.

(39) Castañeda, R.; Corma, A.; Fornés, V.; Martínez-Triguero, J.; Valencia, S. J. Catal. 2006, 238, 79.

(40) Corma, A.; Diaz-Cabanas, M. J.; Jorda, J. L.; Rey, F.; Sastre, G.; Strohmaier, K. G. J. Am. Chem. Soc. 2008, 130, 16482.

(41) Millini, R.; Perego, G.; Parker Jr., W. O.; Bellussi, G.; Carluccio, L. Micropor. Mater. 1995, 4, 221.

(42) Marler, B.; Camblor, M. A.; Gies, H. Micropor. Mesopor. Mater. 2006, 87.

(43) Ikeda, T.; Oumi, Y.; Akiyama, Y.; Kawai, A.; Mizukami, F. Angew. Chem., Int. Ed. 2004, 43, 4982.

(44) Zanardi, S.; Alberti, A.; Cruciani, G.; Corma, A.; Fornés, V.; Brunelli, M. Angew. Chem., Int. Ed. 2004, 43, 4933.

(45) Ikeda, T.; Oumi, Y.; Takeoka, T.; Yokoyama, T.; Sano, T.; Hanaoka, T. Micropor. Mesopor. Mater. 2008, 110, 488.

(46) Wang, Y.; Gies, H.; Marler, B.; Müller, U. Chem. Mater. 2005, 1743. 
(47) Tijsebaert, B.; Varszegi, C.; Gies, H.; Xiao, F. S.; Bao, X.; Tatsumi, T.; Muller, U.; De Vos, D. Chem. Commun. 2008, 2480.

(48) Yilmaz, B.; Muller, U.; Tijsebaert, B.; De Vos, D.; Xie, B.; Xiao, F. S.; Gies, H.; Zhang, W.; Bao, X.; Imai, H.; Tatsumi, T. Chem. Commun. 2011, 47, 1812.

(49) Tijsebaert, B.; Yilmaz, B.; Muller, U.; Gies, H.; Zhang, W.; Bao, X.; Xiao, F. S.; Tatsumi, T.; De Vos, D. J. Catal. 2011, 278, 246.

(50) Corbin, D. R.; Schwarz, S.; Sonnichsen, G. C. Catal. Today 1997, 37, 71.

(51) Roth, W. J.; Nachtigall, P.; Morris, R. E.; Wheatley, P. S.; Seymour, V. R.; Ashbrook, S. E.; Chlubná, P.; Grajciar, L.; Položij, M.; Zukal, A.; Shvets, O.; Čejka, J. Nat. Chem. 2013, 5, 628.

(52) Dumont, N.; Gabelica, Z.; Derouane, E. G.; McCusker, L. B. Micropor. Mater. 1993, 1, 149.

(53) Lourenço, J. P.; Ribeiro, M. F.; Ribeiro, F. R.; Rocha, J.; Gabelica, Z.; Dumont, N.; Derouane, E. G. Stud. Surf. Sci. Catal. 1994, 84, 867.

(54) Lourenço, J. P.; Ribeiro, M. F.; Ribeiro, F. R.; Rocha, J.; Gabelica, Z. Appl. Catal. A 1996, $148,167$.

(55) Lourenço, J. P.; Ribeiro, M. F.; Ribeiro, F. R.; Rocha, J.; Gabelica, Z. React. Kinet. Catal. Lett. 1996, 59, 219.

(56) Morris, R. E.; Burton, A.; Bull, L. M.; Zones, S. I. Chem. Mater. 2004, 16, 2844.

(57) Cao, G.; Afeworki, M.; Kennedy, G. J.; Strohmaier, K. G.; Dorset, D. L. Acta Crystal. B 2007, 63, 56.

(58) Akolekar, D. B. Zeolites 1995, 15, 583.

(59) Patinec, V.; Wright, P. A.; Lightfoot, P.; Aitken, R. A.; Cox, P. A. J. Chem. Soc., Dalton Trans. 1999, 3909.

(60) Bu, X.; Feng, P.; Stucky, G. D. Science 1997, 278, 2080.

(61) Blackwell, C. S.; Broach, R. W.; Gatter, M. G.; Holmgren, J. S.; Jan, D. Y.; Lewis, G. J.; Mezza, B. J.; Mezza, T. M.; Miller, M. A.; Moscoso, J. G.; Patton, R. L.; Rohde, L. M.; Schoonover, M. W.; Sinkler, W.; Wilson, B. A.; Wilson, S. T. Angew. Chem., Int. Ed. 2003, 42, 1737.

(62) Higgins, J. B.; Schmitt, K. D. Zeolites 1996, 16, 236.

(63) Itakura, M.; Oumi, Y.; Sadakane, M.; Sano, T. Mater. Resear. Bull. 2010, 45, 646.

(64) (a) Breck, D. W.; Acara, N. A. U.S.Patent 2991151, 1961. (b) Andries, K. J.; Bosmans, H. J.; Grobet, P. J. Zeolites 1991, 11, 124.

(65) Monteki, T.; Okubo, T. Chem. Mater. 2013, 25, 2603.

(66) Ciric, J. U.S.Patent 3692470, 1972.

(67) (a) Aiello, R.; Barrer, R. M.; Davies, J. A.; Kerr, I. S. Trans. Faraday Soc. 1970, 66, 1610.

(b) Moudafi, L.; Dutarte, R.; Fajula, F.; Figueras, F. Appl. Catal. 1986, 20, 189. (c) Matijasic, A.; Patarin, J. Micropor. Mesopor. Mater. 1999, 29, 405.

(68) (a) Sastre, E.; Corma, A.; Fajula, F.; Figueras, F.; Perez-Pariente, J. J. Catal. 1990, 126, 457. (b) Martens, J. A.; Perez-Pariente, J.; Sastre, E.; Corma, A.; Jacobs, P. A. Appl. Catal. 1988, 45, 85.

(69) (a) Arous, W.; Tounsi, H.; Djemel, S.; Ghorbel, A.; Delahay, G. Top. Catal. 2007, 42-43, 51. (b) Arous, W.; Tounsi, H.; Djemel, S.; Ghorbel, A.; Delahay, G. Catal. Commun. 2005, 6, 281.

(70) Chen, N. Y.; Schlenker, J. L.; Garwood, W. E.; Kokotailo, G. T. J. Catal. 1984, 86, 24.

(71) Hernandez, F.; Moudafi, L.; Fajula, F.; Figueras, F. Proceed. 8th Int. Congr. Catal. 1985, $2,1447$.

(72) Dejaifve, P.; Auroux, A.; Gravelle, P. C.; Vedrine, J. C.; Gabelica, Z.; Derouane, E. G. J. Catal. 1981, 70, 123.

(73) Meier, W. M. Z. Kristallogr. 1961, 115, 439. 
(74) (a) Fernandes, L. D.; Monteiro, J. L. F.; Sousa-Aguiar, E. F.; Martinez, A.; Corma, A. J. Catal. 1998, 177, 363. (b) Sasaki, H.; Oumi, Y.; Itabashi, K.; Lu, B.; Teranishi, T.; Sano, T. J. Mater. Chem. 2003, 13, 1173.

(75) (a) Hamidi, F.; Bengueddach, A.; Di Renzo, F.; Fajula, F. Catal. Lett. 2003, 87, 149. (b) Sharma, P.; Rajaram, P.; Tomar, R. J. Colloid Inter. Sci. 2008, 325, 547. (c) Zhang, L.; van Laak, A. N. C.; de Jongh, P. E.; De Jong, K. P. Micropor. Mesopor. Mater. 2009, 126, 115. (76) Zhang, L.; Xie, S.; Xin, W.; Li, X.; Liu, S.; Xu, L. Mater. Resear. Bull. 2011, 46, 894.

(77) Kato, M.; Itabashi, K.; Matsumoto, A.; Tsutsumi, K. J. Phys. Chem. B 2003, 107, 1788.

(78) (a) Bajpai, P. K.; Rao, M. S.; Gokhale, K. V. G. K. Ind. Eng. Chem. Prod. Rd. 1978, 17, 223. (b) Groothaert, M. H.; Smeets, P. J.; Sels, B. F.; Jacobs, P. A.; Schoonheydt, R. A. J. Am. Chem. Soc. 2005, 127, 1394. (c) van Donk, S.; Broersma, A.; Gijzeman, O. L. J.; van Bokhoven, J. A.; Bitter, J. H.; de Jong, K. P. J. Catal. 2001, 204, 272. (d) Samata, S.; Mal, N. K.; Kumar, P.; Bhaumik, A. J. Mol. Catal. A 2004, 215, 169. (e) Corma, A.; Frontela, J.; Lazaro, J.; Perez, M. Preprints - American Chemical Society, Division of Petroleum Chemistry 1991, 36, 833.

(79) Lazaro, J.; Corma, A.; Frontela, J. US 5,057,471 (1991).

(80) Magnoux, P.; Guisnet, M. Appl. Catal. 1988, 38, 341.

(81) (a) Smith, W. J. EP 0,596,632 (1993). (b) Ellis, B.; Howard, M. J.; Joyner, R. W.; Reddy, K. N.; Padley, M. B.; Smith, W. J. Stud. Surf. Sci. Catal. 1996, 101, 771. (c) Cheung, P.; Bhan, A.; Sunley, G. J.; Iglesia, E. Angew. Chem., Int. Ed. 2006, 45, 1617.

(82) Sunley, G. J.; Watson, D. J. Catal. Today. 2000, 58, 293.

(83) Pluth, J. J.; Smith, J. V. Am. Miner. 1990, 75, 501.

(84) Willhammar, T.; Sun, J.; Wan, W.; Olevnikov, P.; Zhang, D.; Zou, X.; Moliner, M.; Gonzalez, J.; Martínez, C.; Rey, F.; Corma, A. Nature Chem. 2012, 4, 188.

(85) Lobo, R. F.; Pan, M.; Chan, I.; Li, H. X.; Medrud, R. C.; Zones, S. I.; Crozier, P. A.; Davis, M. E. Science 1993, 262, 1543.

(86) Lobo, R. F.; Pan, M.; Chan, I.; Medrud, R. C.; Zones, S. I.; Crozier, P. A.; Davis, M. E. J. Phys. Chem. 1994, 98, 12040.

(87) Lobo, R. F.; Davis, M. E. J. Am. Chem. Soc. 1995, 117, 3766.

(88) (a) Corma, A.; Navarro, M. T.; Rey, F.; Rius, J.; Valencia, S. Angew. Chem., Int. Ed. 2001, 40, 2277. (b) Corma, A.; Navarro, M. T.; Rey, F.; Valencia, S. Chem. Commun. 2001, 1486. (c) Sastre, G.; Vidal-Moya, A.; Blasco, T.; Rius, J.; Jorda, J. L.; Navarro, M. T.; Rey, F.; Corma, A. Angew. Chem., Int. Ed. 2002, 41, 4722. (d) Blasco, T.; Corma, A.; DiazCabañas, M. J.; Rey, F.; Vidal-Moya, A.; Zicovich-Wilson, C. M. J. Phys. Chem. B 2002, 106, 2637.

(89) Castaneda, R.; Corma, A.; Fornes, V.; Rey, F.; Rius, J. J. Am. Chem. Soc. 2003, 125, 7820.

(90) Cantín, A.; Corma, A.; Diaz-Cabanas, M. J.; Jorda, J. L.; Moliner, M. J. Am. Chem. Soc. 2006, 128, 4216.

(91) (a) Adair, B.; Chen, C. Y.; Wan, K. T.; Davis, M. E. Micropor. Mater. 1996, 7, 261. (b) Jones, C. W.; Zones, S. I.; Davis, M. E. Micropor. Mesopor. Mater. 1999, 28, 471.

(92) Corma, A.; Corell, C.; Llopis, F. J.; Martinez, A.; Perez-Pariente, J. Appl. Catal. A 1994, $115,121$.

(93) (a) Corma, A. Micropor. Mesopor. Mater. 1998, 21, 487. (b) Sastre, G.; Corma, A.; Catlow, R. A. Top. Catal. 1999, 9, 215.

(94) Galli, E.; Quartieri, S.; Vezzalini, G.; Alberti, A. Eur. J. Mineral 1995, 7, 1029.

(95) Simancas, R.; Dari, D.; Velamazán, N.; Navarro, M. T.; Cantín, A.; Jordá, J. L.; Sastre, G.; Corma, A.; Rey, F. Science 2010, 330, 1219.

(96) Dorset, D. L.; Weston, S. C.; Dhingra, S. S. J. Phys. Chem. B 2006, 110, 2045.

(97) Shibata, T.; Suzuki, S.; Kawagoe, H.; Komura, K.; Kubota, Y.; Sugi, Y.; Kim, J. H.; Seo, G. Micropor. Mesopor. Mater. 2008, 116, 216.

(98) Shibata, T.; Kawagoe, H.; Naiki, H.; Komura, N.; Kubota, Y.; Sugi, Y. J. Mol. Catal. A 2009, 297, 80 . 
(99) Sugi, Y.; Maekawa, H.; Hasegawa, Y.; Ito, A.; Asai, R.; Yamamoto, D.; Komura, K.; Kubota, Y.; Kim, J. H.; Seo, G. Catal. Today 2008, 131, 13.

(100) Inagaki, S.; Takechi, K.; Kubota, Y. Chem. Commun. 2010, 46, 2662.

(101) Kubota, Y.; Koyama, Y.; Yamada, T.; Inagaki, S.; Tatsumi, T. Chem. Commun. 2008, 6224.

(102) Elomari, S.; Burton, A.; Medrud, R. C.; Grosse-Kunstleve, R. Micropor. Mesopor. Mater. 2009, 118, 325.

(103) Xie, D.; McCusker, L. B.; Baerlocher, C. J. Am. Chem. Soc., 133, 20604.

(104) Burton, A. W. U.S.Patent 7820141, 2010.

(105) Baerlocher, C.; Weber, T.; McCusker, L. B.; Palatinus, L.; Zones, S. I. Science 2011, 333, , 1134.

(106) Zones, S. I.; Ruan, J.; Elomari, S.; Terasaki, O.; Chen, C. Y.; Corma, A. Solid State Sci. 2011, 13, 706.

(107) Chen, C. Y.; Zones, S. I.; Huang, S. J.; Bull, L. M. Stud. Surf. Sci. Catal. 2004, 154B, 1547.

(108) Zones, S. I.; Chen, C. Y.; Benin, A.; Hwang, S. J. J. Catal. 2013, 308, 213.

(109) Zones, S. I.; Benin, A.; Hwang, S. J.; Xie, D.; Elomari, S.; Hsieh, M. F. J. Am. Chem. Soc. 136, 136, 1462.

(110) Corma, A.; Rey, F.; Valencia, S.; Jorda, J. L.; Rius, J. Nature Mater. 2003, 2, 493.

(111) (a) Burel, L.; Kasian, N.; Tuel, A. Angew. Chem., Int. Ed. 2014, 53, 1360. (b) ChlubnaEliasova, P.; Tian, Y.; Pinar, A. B.; Kubu, M.; Cejka, J.; Morris, R. E. Angew. Chem., Int. Ed. 2014, 53, 7048.

(112) Moliner, M.; Rey, F.; Corma, A. Angew. Chem., Int. Ed. 2013, 52, 13880.

(113) Moliner, M.; González, J.; Portilla, M. T.; Willhammar, T.; Rey, F.; Llopis, F. J.; Zou, X.; Corma, A. J. Am. Chem. Soc. 2011, 133, 9497.

(114) Moliner, M.; Corma, A. Micropor. Mesopor. Mater. 2012, 164, 44.

(115) Moliner, M.; Willhammar, T.; Wan, W.; González, J.; Rey, F.; Jorda, J. L.; Zou, X.; Corma, A. J. Am. Chem. Soc. 2012, 1346473.

(116) Chereyeck, L.; Caullet, P. H.; Mougenel, J. C.; Guth, J. L.; Marler, B. Micropor. Mater. 1996, 6, 259.

(117) Ikeda, T.; Kayamori, S.; Oumi, Y.; Mizukami, F. J. Phys. Chem. C 2010, 114, 3466.

(118) Verheyen, E.; Joos, L.; Van Havenbergh, K.; Breynaert, E.; Kasian, N.; Gobechiya, E.; Houthoofd, K.; Martineau, C.; Hinterstein, M.; Taulelle, F.; Van Speybroeck, V.; Waroquier, M.; Bals, S.; Van Tendeloo, G.; Kirschhock, C. E. A.; Martens, J. A. Nat. Mater. 2012, 11, 1059.

(119) (a) Corma, A.; Diaz-Cabañas, M. J.; Rey, F.; Nicolopoulus, S.; Boulahya, K. Chem. Commun. 2004, 1356. (b) Paillaud, J. L.; Harbuzaru, B.; Patarin, J.; Bats, N. Science 2004, 304, 990.

(120) Jiang, J.; Yu, J.; Corma, A. Angew. Chem., Int. Ed. 2010, 49, 3120.

(121) Blasco, T.; Corma, A.; Díaz-Cabañas, M. J.; Rey, F.; Vidal-Moya, A.; Zicovich-Wilson, C. M. J. Phys. Chem. B 2002 2002, 106, 2637.

(122) Brunner, G. O.; Meier, W. M. Nature 1989, 337, 146.

(123) (a) Corma, A.; Díaz-Cabañas, M. J.; Jordá, J. L.; Martínez, C.; Moliner, M. Nature 2006, 443, 842. (b) Sun, J.; Bonneau, C.; Cantín, A.; Corma, A.; Diaz-Cabañas, M. J.; Moliner, M.; Zhang, D.; Li, M.; Zou, X. Nature 2009, 458, 1154. (c) Jiang, J.; Jorda, J. L.; DiazCabañas, M. J.; Yu, J.; Corma, A. Angew. Chem., Int. Ed. 2010, 49, 4986. (d) Jiang, J.; Jorda, J. L.; Yu, J.; Baumes, L. A.; Mugnaioli, E.; Diaz-Cabanas, M. J.; Kolb, U.; Corma, A. Science 2011, 333, 1131. (e) Corma, A.; Díaz-Cabañas, M. J.; Jiang, J.; Afeworki, M.; Dorset, D. L.; Soled, S. L.; Strohmaier, K. G. Proc. Natl. Acad. Sci. U.S.A. 2010, 107, 11935.

(124) (a) Corma, A.; Diaz-Cabañas, M. J.; Rey, F. Sp. Pat. ES2186487, 2000. (b) Corma, A.; Diaz-Cabañas, M. J.; Rey, F. PCT Int. Appl. WO0203820, 2002. 
(125) (a) Kasian, N.; Koranyi, T. I.; Vanbutsele, G.; Houthoofd, K.; Martens, J. A.; Kirschhock, C. E. A. Top. Catal. 2010, 53, 1374. (b) Kasian, N.; Vanbutsele, G.; Houthoofd, K.; Koranyi, T. I.; Martens, J. A.; Kirschhock, C. E. A. Catal. Sci. Technol. 2011, 1, 246. (c) Kasian, N.; Verheyen, E.; Vanbutsele, G.; Houthoofd, K.; Koranyi, T. I.; Martens, J. A.; Kirschhock, C. E. A. Micropor. Mesopor. Mater. 2013, 166, 153.

(126) Zilkova, N.; Shamzhy, M.; Shvets, O.; Cejka, J. Catal. Today 2013, 204, 22.

(127) Moliner, M.; Diaz-Cabañas, M. J.; Fornes, V.; Martinez, C.; Corma, A. J. Catal. 2008, 254, 101.

(128) Xieqing, W.; Chaogang, X.; Zaiting, L.; Genquan, Z. "Catalytic Processes for Light Olefin Production", In Practical Advances in Petroleum Processing; Eds: C. S. Hsu, P. R. Robinson; Springer; pp: 149-168 (2006).

(129) (a) Xu, H.; Jiang, J. G.; Yang, B.; Zhang, L.; He, M.; Wu, P. Angew. Chem., Int. Ed. 2014, 53, 1355. (b) Gao, F.; Jaber, M.; Bozhilov, K.; Vicente, A.; Fernandez, C.; Valtchev, V. J. Am. Chem. Soc. 2009, 131, 16580.

(130) Iyoki, K.; Itabashi, K.; Okubo, T. Micropor. Mesopor. Mater. 2014, 189, 22.

(131) Prakash, A. M.; Ashtekar, S.; Chakrabarty, D. K. J. Chem. Soc. Faraday Trans. 1995, 91, 1045.

(132) Chippindale, A. M.; Cowley, A. R. Zeolites 1997, 18, 176.

(133) Cowley, A. R.; Chippindale, A. M. Micropor. Mesopor. Mater. 1999, 28, 163.

(134) Hong, S. B.; Kim, S. H.; Kim, Y. G.; Kim, Y. C.; Barrett, P. A.; Camblor, M. A. J. Mater. Chem. 1999, 9, 2287.

(135) Leonowicz, M. E.; Vaughan, D. E. W. Nature 1987, 329, 819.

(136) Strohmaier, K. G.; Vaughan, D. E. W. J. Am. Chem. Soc. 2003, 125, 16035.

(137) Vaughan, P. A. Acta Crystallogr. 1966, 21, 983.

(138) Schlenker, J. L.; Higgins, J. B.; Valyocsik, E. W. Zeolites 1990, 10, 293.

(139) Howden, M. G. Zeolites 1987, 7, 255.

(140) Lawton, S. L.; Bennett, J. M.; Schlenker, J. L.; Rubin, M. K. Chem. Commun. 1993, 894.

(141) Lorgouilloux, Y.; Dodin, M.; Paillaud, J. L.; Caullet, P.; Michelin, L.; Josien, L.; Ersen, O.; Bats, N. J. Solid State Chem. 2009, 182, 622.

(142) Dodin, M.; Paillaud, J. L.; Lorgouillous, Y.; Caullet, P.; Elkaim, E.; Bats, N. J. Am. Chem. Soc. 2010, 132, 10221. 